Check for updates

Cite this: RSC Adv., 2017, 7, 47507

\title{
Precision polymers containing main-chain-amino acids: ADMET polymerization and crystallization $\uparrow$
}

\author{
Jan Freudenberg, (D) ${ }^{a}$ Silvio Poppe ${ }^{b}$ and Wolfgang H. Binder (D)*a
}

The synthesis of new poly(alkyl)-precision oligomers and polymers displaying different types of amino acids (chiral/achiral, polar/non-polar) placed at every $19^{\text {th }}$ carbon atom are presented. Placing $\omega$-alkenyl moieties onto appropriately $\mathrm{N}$ - and $\mathrm{C}$-terminus functionalized amino acids successfully enabled ADMETpolymerization of these monomers. Grubbs $1^{\text {st }}$ catalyst under melt-polycondensation-conditions was found to be the most effective with respect to the obtained molecular weights of the polymers, the isomerization of the olefins and their final yield, yielding molecular weights up to $\sim 22 \mathrm{kDa}$. The obtained polymers and the subsequent hydrogenation of the double bonds with $p$-toluenesulfonhydrazide $\left(\mathrm{TsNHNH}_{2}\right.$ ) was proven by NMR, GPC, MALDI-ToF-MS and IR measurements. Investigation of the thermal behavior of the monomers and polymers via DSC measurements displays amorphous structures for monomers and polymers with unpolar amino acid side chains, whereas for polymers bearing the polar glutamic- and aspartic acid moieties crystalline morphologies are observed. An ordered lamellar crystal phase is observed where the amino acid branches are either incorporated or excluded from the unit cell, as proven by WAXS data.

Received 2nd September 2017 Accepted 29th September 2017

DOI: 10.1039/c7ra10485e

rsc.li/rsc-advances engineered by repetitively placing monomers at specific positions within a polymer chain of defined origin. ${ }^{\mathbf{1 0 - 1 5}}$ Acyclic diene metathesis polymerization (ADMET) ${ }^{\mathbf{1 6}}$ has been proven to be an excellent tool to achieve a polyethylene (PE)-like chain with functional monomers at precise locations. ${ }^{17,18}$ Size, chemical structure and the steric structure of such "defects" to a large extent determine the final crystallization behaviour in the solid state: when the precisely placed chemical elements are small (such as methyl ${ }^{19,20}$-, ethyl ${ }^{21}$ - or halogen ${ }^{22-26}$-groups) they can be incorporated into the crystalline region of a semicrystalline polymer, whereas larger defects (higher alkyl substituents, ${ }^{19,27}$ phosphoesters $^{\mathbf{2 8 , 2 9}}$ or triazol-rings ${ }^{30}$ and sulfonic-moieties ${ }^{31}$ ) are excluded from the final crystal-lattice.

The synthetic preparation of biosimilar oligomers and polymers with repetitive amino acid units in the main chains has stimulated a plethora of different methods. ${ }^{32,33}$ Thus e.g. the synthesis of optically active linear and branched poly(meth) acrylamides bearing L-amino acid moieties by radical polymerization $^{34-37}$ or the periodically incorporation of amino acid units via ROMP using Grubbs catalysts has been accomplished. ${ }^{38-40}$ Especially ROMP was found to be an excellent tool for synthesizing polyolefin-graft-oligopeptide polyelectrolytes which can form extended, pearl-like, or multi-molecular structures. ${ }^{\mathbf{4 1}} \mathrm{A}$ periodic arrangement of amino acid branches in precision polymers via ADMET polymerization has been previously achieved allowing to prepare polyolefines bearing ester-terminated and amide-terminated amino acids in the backbone. ${ }^{42}$ Currently, only lysine as a side-chain moiety and cysteine (within the main chain) have been placed within a poly(alkyl)-
${ }^{a}$ Martin Luther University Halle-Wittenberg, Faculty of Natural Sciences II, Chair of Macromolecular Chemistry, D-06120 Halle, Germany

${ }^{b}$ Martin Luther University Halle-Wittenberg, Faculty of Natural Sciences II, Chair of Organic Chemistry, D-06120 Halle, Germany

$\dagger$ Electronic supplementary information (ESI) available: ${ }^{1} \mathrm{H}$ and ${ }^{13} \mathrm{C}$ NMR data of monomers as well as ${ }^{1} \mathrm{H}$ - and ${ }^{13} \mathrm{C}-\mathrm{NMR}$-, IR-data and MALDI-ToF-MS-data of polymers. See DOI: 10.1039/c7ra10485e 

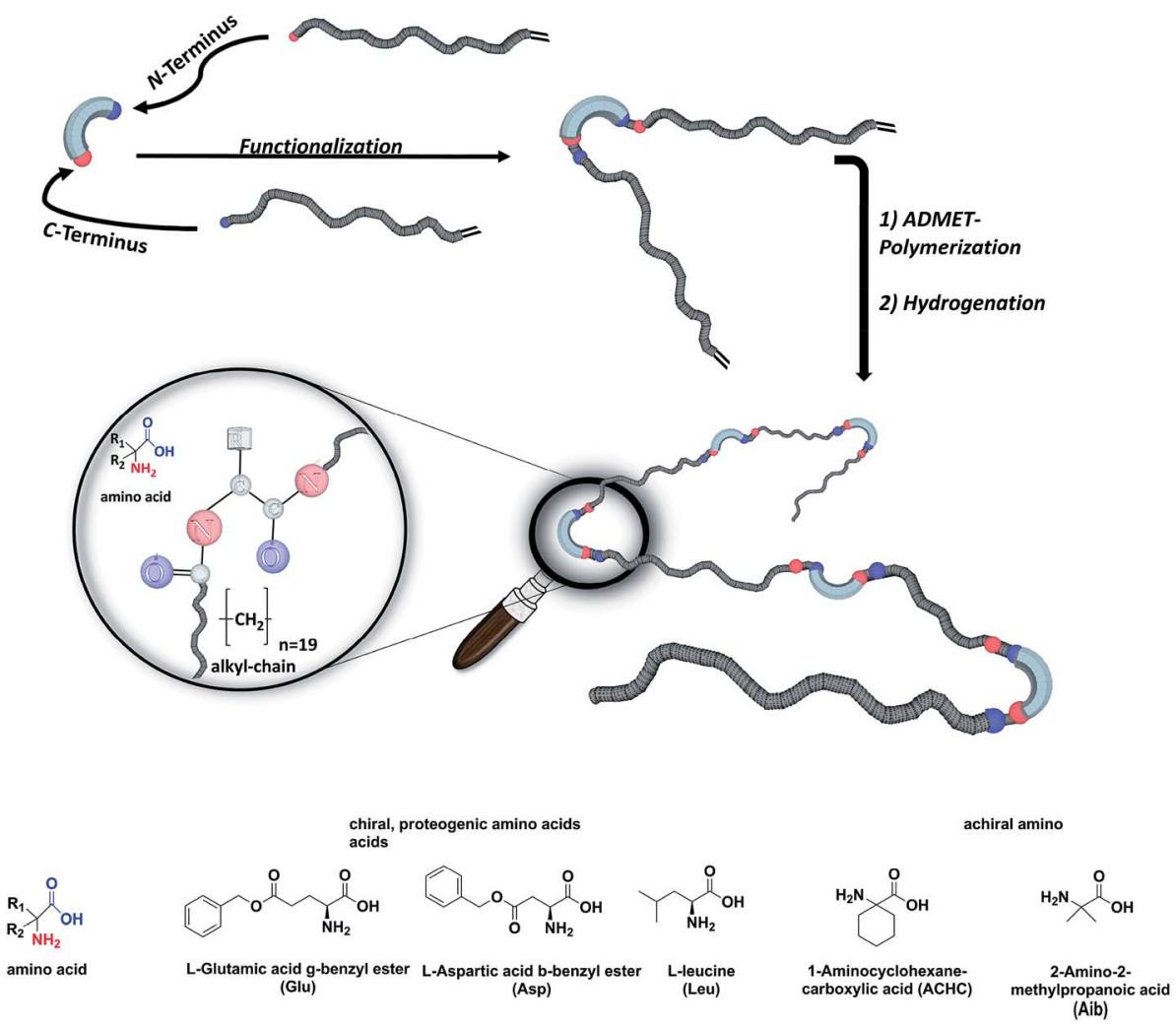

Fig. 1 Schematic representation of the synthesis of PE precision polymers with amino acid defects.

chain $^{\mathbf{4 3 , 4 4}}$ resulting in a semi-crystalline morphology of such polymers.

We here report on the introduction of five different aminoacids (chiral/achiral, polar/non-polar) as basic bio-building blocks into the main chain of PE-polymers, placed precisely at every $19^{\text {th }}$ carbon within the main chain by functionalizing the $N$ - and $C$-terminus of the amino acid (see Fig. 1). Our main interest concerned the synthetic/preparative issue, most of all how ADMET will comply with different amino acids to furnish after hydrogenation - the final polymers with precisely placed amino acids. On the other hand we were interested in the structure formation of such polymers in the solid (semicrystalline) state: which amino acid will allow crystallization of the poly(alkyl)-chain, which one will inhibit the crystal-formation, and what will happen to the crystal structure if formed.

\section{Experimental section}

\section{Materials}

Amino acids L-glutamic acid $\gamma$-benzyl ester and $\mathrm{L}$-aspartic acid $\beta$-benzyl ester were purchased from carbolution-chemicals, 1-aminocyclohexanecarboxylic acid, 2-aminoisobutyric acid and $\mathrm{LiAlH}_{4}$ were purchased from ABCR and 10-undecenoyl chloride was purchased from Acros Organics. All other chemicals were purchased from Sigma-Aldrich. All chemicals were used without further preparations or purification steps. The solvents which were used for reactions were dried and freshly distilled before using. THF was dried and distilled over sodium and benzophenone under $\mathrm{N}_{2}$ atmosphere, for drying of dichloromethane (DCM) and dimethyl formamide (DMF) $\mathrm{CaH}_{2}$ was used. For the reactions under protective gas atmosphere nitrogen $\left(\mathrm{N}_{2}\right)$ was used, which was purchased from LINDE.

\section{Instrument and measurements}

Nuclear magnetic resonance (NMR) spectra were recorded at $27^{\circ} \mathrm{C}$ on a NMR spectrometer of VARIAN. The ${ }^{1} \mathrm{H}$-NMR spectroscopy were performed at $400 \mathrm{MHz}$ and ${ }^{13} \mathrm{C}$-NMR spectroscopy at 100 MHz. For all samples $\mathrm{CDCl}_{3}$ or DMSO- $\mathrm{d}_{6}$ were used as solvents, in case of the hydrogenated polymers $4 \mathbf{4 a}-\mathbf{4 e} 15$ vol\% TFA were added. The splitting patterns were subdivided in singuletts (s), dubletts (d), triplets ( $\mathrm{t}$ ) and multiplett $(\mathrm{m})$ during analysis. Chemical shifts $(\delta)$ were given in $\mathrm{ppm}$ referred to the solvent residue peak and tetramethylsilane were used as internal standard.

Infrared-spectroscopy (IR) was measured by VERTEX 70 IR spectrometer of Bruker by using a single reflex-diamond-ATRunit. The absorption bands are reported in $\mathrm{cm}^{-1}$ in an area of $400-4000 \mathrm{~cm}^{-1}$. The software OPUS was used for interpretation of the obtained bands.

Matrix-assisted laser desorption/ionization time-of-flight mass spectrometry (MALDI-ToF-MS) measurements were performed on a Bruker Autoflex III system (Bruker Daltonics) using a nitrogen laser operating at a wave length of $\lambda=337 \mathrm{~nm}$ in reflection or linear modes. The polymer was dissolved in THF with a concentration of $10 \mathrm{mg} \mathrm{mL} \mathrm{m}^{-1}$. The matrix dithranol was dissolved in THF with a concentration of $10 \mathrm{mg} \mathrm{mL}^{-1}$, for the salt KTFA a concentration of $5 \mathrm{mg} \mathrm{mL}^{-1}$ in THF was used. The 
volume ratio of the components was matrix : analyt : salt was for all measurements $100: 10: 1$. Calibration was done with PEG-standard $\left(M_{\mathrm{p}}=2000 \mathrm{~g} \mathrm{~mol}^{-1}\right.$ and $\left.4200 \mathrm{~g} \mathrm{~mol}^{-1}\right)$. Data evaluation was carried out on flexAnalysis software (3.4).

For gel permeation chromatography (GPC) measurements a Viscotek GPCmax VE 2002 with a HHR-HGuard-17369 and a GMH HR-N-18055 column in tetrahydrofuran (THF) was used. Measurements were done at $22{ }^{\circ} \mathrm{C}$ and concentration of the samples were $c=5 \mathrm{mg} \mathrm{ml}^{-1}$. Detection was realized by refractive index with a VE3580 RI detector of Viscotek at a temperature

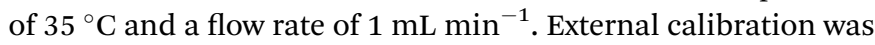
done with polystyrene $\left(M_{\mathrm{p}}=1000-115000 \mathrm{~g} \mathrm{~mol}^{-1}\right)$.

For differential scanning calorimetry (DSC) measurements a NETZSCH DC 204F1 Phoenix, which was calibrated with indium, tin, bismuth, and zinc was used. Nitrogen was used as purge gas and all samples $(3 \mathrm{~g}-8 \mathrm{~g})$ were placed in standard aluminum pans. For analysis of obtained data Netzsch Proteus - Thermal Analysis (version 5.2.1.) and OriginPro 8G was used. For calculation of the crystallinity $\alpha$ equation below was used:

$$
\alpha=\frac{\Delta H_{\mathrm{m}}}{\Delta H_{\mathrm{m}}^{0}} \times 100
$$

where $H_{\mathrm{m}}^{0}$ is the fusion enthalpy of eicosane $\Delta H_{\mathrm{m}}=247.3 \mathrm{~J} \mathrm{~g}^{-1}$.

$\mathrm{X}$-ray diffraction (XRD) 2D patterns were detected on Vantec 500 detector (Bruker AXS) with a detector to sample distance of $9.00 \mathrm{~cm}$. Measurements of the samples were done as droplet which was heated up on a glass plate to isotropic state followed by cooling the sample with a define cooling rate of $5 \mathrm{~K} \mathrm{~min}^{-1}$ on a temperature-controlled heating stage. Measuring temperature for $4 \mathrm{a}$ was $60.0^{\circ} \mathrm{C}$ and for $4 \mathbf{b} 70.0^{\circ} \mathrm{C}$, measuring time for both samples were $30 \mathrm{~min}$.

Dialysis tube (MWCO: $1000 \mathrm{~g} \mathrm{~mol}^{-1}$ ) was purchased from Carl Roth GmbH.

\section{Monomer synthesis}

\section{$\boldsymbol{N}$-terminus functionalization}

General procedure for the amino acids bearing a benzyl protection group in the side chain (method A) $(\mathbf{1 a - 1 b}) . N$-terminus functionalization was realized by the Einhorn method for the amino acids bearing a benzyl protection group in the side chain. Therefore, the protected amino acid (1 eq.) was dissolved in THF $(12 \mathrm{~mL})$ and pyridine $(10 \mathrm{~mL})$ was added to the solution. The mixture was cooled to $0{ }^{\circ} \mathrm{C}$ and 10 -undecenoyl chloride (1.3 eq.) was added dropwise to the solution, which then was stirred for $24 \mathrm{~h}$ at room temperature. Then the reaction mixture was poured into cold $1 \mathrm{M} \mathrm{HCl}$-solution $(300 \mathrm{~mL})$ and diethyl ether $(200 \mathrm{~mL})$ was added. The organic phase were separated, extracted two times with $1 \mathrm{M} \mathrm{HCl}$ solution $(2 \times 100 \mathrm{~mL})$ and dried over $\mathrm{Na}_{2} \mathrm{SO}_{4}$. After filtration the mixture was concentrated in vacuum and recrystallized three times in ethyl acetate $: n$-hexane (1 : 40) mixture to yield a white solid 1a-1b (72-76\%).

$N$-Glu (1a): ${ }^{1} \mathrm{H}-\mathrm{NMR}\left(\mathrm{CDCl}_{3}, 27{ }^{\circ} \mathrm{C}, 400 \mathrm{MHz}\right): \delta[\mathrm{ppm}] 1.27$ $\left(\mathrm{m}, 10 \mathrm{H}, H_{\mathrm{d}}-H_{\mathrm{h}}\right), 1.60\left(\mathrm{~m}, 2 \mathrm{H}, H_{\mathrm{i}}\right), 2.02-2.20\left(\mathrm{~m}, 6 \mathrm{H}, H_{4}+H_{\mathrm{c}}+\right.$ $\left.H_{\mathrm{j}}\right), 2.49\left(\mathrm{~m}, 1 \mathrm{H}, H_{3}\right), 2.57\left(\mathrm{~m}, 1 \mathrm{H}, H_{3}\right), 4.54\left(\mathrm{~m}, 1 \mathrm{H}, H_{2}\right), 4.93(\mathrm{~m}$, $\left.2 \mathrm{H}, H_{\mathrm{a}}\right), 5.12\left(\mathrm{~s}, 2 \mathrm{H}, H_{6}\right), 5.79\left(\mathrm{~m}, 1 \mathrm{H}, H_{\mathrm{b}}\right), 6.56\left(\mathrm{~d},{ }^{3} J_{\mathrm{H}, \mathrm{H}}=7.2 \mathrm{~Hz}\right.$, $\left.1 \mathrm{H}, H_{13}\right), 7.31-7.45\left(\mathrm{~m}, 5 \mathrm{H}, H_{8}-H_{12}\right)$.
$N$-Glu (1a): ${ }^{13} \mathrm{C}-\mathrm{NMR}\left(\mathrm{CDCl}_{3}, 27{ }^{\circ} \mathrm{C}, 100 \mathrm{MHz}\right): \delta[\mathrm{ppm}] 25.4$ $\left(C_{\mathrm{i}}\right), 26.5\left(C_{3}\right), 28.9-29.5\left(C_{\mathrm{d}}-C_{\mathrm{h}}\right), 30.5\left(C_{4}\right), 33.7\left(C_{\mathrm{c}}\right), 36.3\left(C_{\mathrm{j}}\right)$, $52.3\left(C_{2}\right), 66.9\left(C_{6}\right), 114.1\left(C_{\mathrm{a}}\right), 128.2-128.7\left(C_{8}-C_{12}\right), 135.5\left(C_{7}\right)$, $139.1\left(C_{\mathrm{b}}\right), 171.5\left(C_{1}\right), 173.6\left(C_{5}\right), 174.1\left(C_{\mathrm{k}}\right)$.

$N$-Asp (1b): ${ }^{1} \mathrm{H}-\mathrm{NMR}\left(\mathrm{CDCl}_{3}, 27{ }^{\circ} \mathrm{C}, 400 \mathrm{MHz}\right): \delta[\mathrm{ppm}] 1.27$ $\left(\mathrm{m}, 10 \mathrm{H}, H_{\mathrm{d}}-H_{\mathrm{h}}\right), 1.61\left(\mathrm{~m}, 2 \mathrm{H}, H_{\mathrm{i}}\right), 2.02\left(\mathrm{~m}, 2 \mathrm{H}, H_{\mathrm{c}}\right), 2.20(\mathrm{~m}$, $\left.2 \mathrm{H}, H_{\mathrm{j}}\right), 2.92\left(\mathrm{~m}, 1 \mathrm{H}, H_{3}\right), 3.09\left(\mathrm{~m}, 1 \mathrm{H}, H_{3}\right), 4.91\left(\mathrm{~m}, 1 \mathrm{H}, H_{2}\right), 4.96$ $\left(\mathrm{m}, 2 \mathrm{H}, H_{\mathrm{a}}\right), 5.14\left(\mathrm{~s}, 2 \mathrm{H}, H_{5}\right), 5.81\left(\mathrm{~m}, 1 \mathrm{H}, H_{\mathrm{b}}\right), 6.56\left(\mathrm{~d},{ }^{3} J_{\mathrm{H}, \mathrm{H}}=\right.$ $\left.7.2 \mathrm{~Hz}, 1 \mathrm{H}, H_{12}\right), 7.31-7.45\left(\mathrm{~m}, 5 \mathrm{H}, H_{7}-H_{11}\right)$.

$N$-Asp (1b): ${ }^{13} \mathrm{C}-\mathrm{NMR}\left(\mathrm{CDCl}_{3}, 27{ }^{\circ} \mathrm{C}, 100 \mathrm{MHz}\right): \delta[\mathrm{ppm}] 25.4$ $\left(C_{\mathrm{i}}\right), 28.9-29.3\left(C_{\mathrm{d}}-C_{\mathrm{h}}\right), 33.8\left(C_{\mathrm{c}}\right), 36.3\left(C_{\mathrm{j}}\right), 36.5\left(C_{3}\right), 48.5\left(C_{2}\right)$, $67.0\left(C_{5}\right), 114.1\left(C_{\mathrm{a}}\right), 128.2-128.6\left(C_{7}-C_{11}\right), 135.23\left(C_{6}\right), 139.1$ $\left(C_{\mathrm{b}}\right), 171.1\left(C_{1}\right), 173.8\left(C_{4}\right), 174.1\left(C_{\mathrm{k}}\right)$.

General procedure for the amino acids without a protection group in the side chain (method B) $(\mathbf{1 c - 1 e}) . N$-terminus functionalization was realized by Schotten-Baumann synthesis for the water soluble amino acids. The amino acid (1 eq.) and $\mathrm{NaOH}$ ( 2 eq.) was dissolved in water $(20 \mathrm{~mL})$ and the mixture was cooled in an ice-bath. 10-Undecenoyl chloride (1.1 eq.), dissolved in dichloromethane $(20 \mathrm{~mL})$, was added dropwise to the solution which was allowed to stir at room temperature for $24 \mathrm{~h}$. The mixture was poured into cold $1 \mathrm{M} \mathrm{HCl}$-solution $(200 \mathrm{~mL})$ and the organic phase were then separated, dried over $\mathrm{Na}_{2} \mathrm{SO}_{4}$, filtrated and concentrated in vacuum. The crude product was recrystallized three times in ethyl acetate : $n$-hexane $(1: 40)$ mixture to yield a white solid 1c-1e (64-75\%).

$N$-Leu (1c): ${ }^{1} \mathrm{H}-\mathrm{NMR}\left(\mathrm{CDCl}_{3}, 27{ }^{\circ} \mathrm{C}, 400 \mathrm{MHz}\right): \delta[\mathrm{ppm}] 0.95$ $\left(\mathrm{m}, 6 \mathrm{H}, H_{5}+H_{6}\right), 1.28\left(\mathrm{~m}, 10 \mathrm{H}, H_{\mathrm{d}}-H_{\mathrm{h}}\right), 1.60-1.71\left(\mathrm{~m}, 5 \mathrm{H}, H_{\mathrm{i}}+\right.$ $\left.H_{3}+H_{4}\right), 2.02\left(\mathrm{~m}, 2 \mathrm{H}, H_{\mathrm{c}}\right), 2.23\left(\mathrm{~m}, 2 \mathrm{H}, H_{\mathrm{j}}\right), 4.61\left(\mathrm{~m}, 1 \mathrm{H}, H_{2}\right), 4.94$ $\left(\mathrm{m}, 2 \mathrm{H}, H_{\mathrm{a}}\right), 5.79\left(\mathrm{~m}, 1 \mathrm{H}, H_{\mathrm{b}}\right), 5.96\left(\mathrm{~m}, 1 \mathrm{H}, H_{7}\right)$.

$N$-Leu (1c): ${ }^{13} \mathrm{C}-\mathrm{NMR}\left(\mathrm{CDCl}_{3}, 27{ }^{\circ} \mathrm{C}, 100 \mathrm{MHz}\right): \delta[\mathrm{ppm}] 21.9-$ $22.8\left(C_{5}+C_{6}\right), 24.9\left(C_{4}\right), 25.5\left(C_{\mathrm{i}}\right), 28.9-29.5\left(C_{\mathrm{d}}-C_{\mathrm{h}}\right), 33.7\left(C_{\mathrm{c}}\right)$, $36.5\left(C_{\mathrm{j}}\right), 41.1\left(C_{3}\right), 50.8\left(C_{2}\right), 114.1\left(C_{\mathrm{a}}\right), 139.1\left(C_{\mathrm{b}}\right), 173.9\left(C_{1}\right)$, $176.3\left(C_{\mathrm{k}}\right)$.

$N$-Aib (1d): ${ }^{1} \mathrm{H}-\mathrm{NMR}\left(\mathrm{CDCl}_{3}, 27{ }^{\circ} \mathrm{C}, 400 \mathrm{MHz}\right): \delta[\mathrm{ppm}] 1.27-$ $1.36\left(\mathrm{~m}, 10 \mathrm{H}, H_{\mathrm{d}}-H_{\mathrm{h}}\right), 1.57-1.62\left(\mathrm{~m}, 8 \mathrm{H}, H_{2}+H_{3}+H_{\mathrm{i}}\right), 2.04(\mathrm{~m}$, $\left.4 \mathrm{H}, H_{\mathrm{c}}\right), 2.20\left(\mathrm{~m}, 2 \mathrm{H}, H_{\mathrm{j}}\right), 4.96\left(\mathrm{~m}, 2 \mathrm{H}, H_{\mathrm{a}}\right), 5.79\left(\mathrm{~m}, 1 \mathrm{H}, H_{\mathrm{b}}\right), 6.02$ $\left(\mathrm{m}, 1 \mathrm{H}, H_{5}\right)$.

$N$-Aib (1d): ${ }^{13} \mathrm{C}-\mathrm{NMR}\left(\mathrm{CDCl}_{3}, 27{ }^{\circ} \mathrm{C}, 100 \mathrm{MHz}\right): \delta[\mathrm{ppm}] 24.9$ $\left(C_{2}+C_{3}\right), 25.5\left(C_{\mathrm{i}}\right), 28.9-29.5\left(C_{\mathrm{d}}-C_{\mathrm{h}}\right), 33.7\left(C_{\mathrm{c}}\right), 36.8\left(C_{\mathrm{j}}\right), 57.0$ $\left(C_{1}\right), 114.1\left(C_{\mathrm{a}}\right), 139.1\left(C_{\mathrm{b}}\right), 174.4\left(C_{\mathrm{k}}\right), 177.2\left(C_{4}\right)$.

$N$-ACHC (1e): ${ }^{1} \mathrm{H}-\mathrm{NMR}\left(\mathrm{CDCl}_{3}, 27{ }^{\circ} \mathrm{C}, 400 \mathrm{MHz}\right): \delta[\mathrm{ppm}]$ 1.29-1.45 (m, 12H, $\left.H_{\mathrm{d}}-H_{\mathrm{i}}\right), 1.66\left(\mathrm{~m}, 6 \mathrm{H}, H_{3}+H_{4}+H_{5}\right), 1.89(\mathrm{~m}$, $\left.2 \mathrm{H}, H_{2}+H_{6}\right), 2.04\left(\mathrm{~m}, 4 \mathrm{H}, H_{\mathrm{c}}+H_{2}+H_{6}\right), 2.26\left(\mathrm{~m}, 2 \mathrm{H}, H_{\mathrm{j}}\right), 4.94$ $\left(\mathrm{m}, 2 \mathrm{H}, H_{\mathrm{a}}\right), 5.69\left(\mathrm{~m}, 1 \mathrm{H}, H_{7}\right), 5.79\left(\mathrm{~m}, 1 \mathrm{H}, H_{\mathrm{b}}\right)$.

$N$-ACHC (1e): ${ }^{13} \mathrm{C}-\mathrm{NMR}\left(\mathrm{CDCl}_{3}, 27{ }^{\circ} \mathrm{C}, 100 \mathrm{MHz}\right): \delta[\mathrm{ppm}] 21.3$ $\left(C_{3}+C_{5}\right), 25.1-25.5\left(C_{\mathrm{i}}+C_{4}\right), 28.9-29.5\left(C_{\mathrm{d}}-C_{\mathrm{h}}\right), 32.0-33.7\left(C_{2}+\right.$ $\left.C_{6}+C_{\mathrm{c}}\right), 36.6\left(C_{\mathrm{j}}\right), 59.7\left(C_{1}\right), 114.1\left(C_{\mathrm{a}}\right), 139.1\left(C_{\mathrm{b}}\right), 175.1\left(C_{\mathrm{k}}\right)$, $176.0\left(C_{4}\right)$.

\section{$\boldsymbol{C}$-terminus functionalization}

General procedure for chiral AA (2a-2c). Reactions were performed under $\mathrm{N}_{2}$ atmosphere. $N$-terminus functionalized amino acid 1a-1c (1.0 eq.) was dissolved in dried dichloromethane $(20 \mathrm{~mL})$ and then $\mathbf{A 2}$ (1.0 eq.) was added to the mixture. The solution was cooled down to $0{ }^{\circ} \mathrm{C}$ and the coupling reagents DIPEA (3.6 eq.), $\mathrm{HOBt}\left(13 \% \mathrm{H}_{2} \mathrm{O} ; 1.6\right.$ eq. $)$ and $\mathrm{EDC} \cdot \mathrm{HCl}$ (1.6 eq.) were added successively. The mixture was allowed to 
stir at room temperature for 4 hours before it was refluxed overnight. Then the solution was cooled and extracted with brine $(20 \mathrm{~mL}), 1 \mathrm{M} \mathrm{HCl}(20 \mathrm{~mL})$, brine $(20 \mathrm{~mL})$, saturated $\mathrm{NaHCO}_{3}$-solution $(20 \mathrm{~mL})$ and brine $(20 \mathrm{~mL})$. The organic phase was concentrated under vacuum and the crude product was dissolved in THF ( $5 \mathrm{~mL})$, precipitated in cold $\mathrm{NaHCO}_{3}(150 \mathrm{~mL})$ solution and isolated by filtration. Afterwards the obtained brown solid was dissolved in $\mathrm{Et}_{2} \mathrm{O}(20 \mathrm{~mL})$, dried over $\mathrm{Na}_{2} \mathrm{SO}_{4}$, filtrated and concentrated in vacuum to yield products $\mathbf{2 a - 2 c}$ $(63-86 \%)$.

$N$ - $+C$-Glu (2a): ${ }^{1} \mathrm{H}-\mathrm{NMR}\left(\mathrm{CDCl}_{3}, 27{ }^{\circ} \mathrm{C}, 400 \mathrm{MHz}\right): \delta[\mathrm{ppm}]$ 1.26-1.34 (m, 20H, $\left.H_{\mathrm{d}}-H_{\mathrm{h}}\right), 1.44-1.60\left(\mathrm{~m}, 4 \mathrm{H}, H_{\mathrm{l}}+H_{\mathrm{i}}\right), 2.03-$ $2.20\left(\mathrm{~m}, 6 \mathrm{H}, H_{4}+H_{\mathrm{c}}+H_{\mathrm{j}}\right), 2.40\left(\mathrm{~m}, 1 \mathrm{H}, H_{3}\right), 2.54\left(\mathrm{~m}, 1 \mathrm{H}, H_{3}\right)$, $3.18\left(\mathrm{~m}, 2 \mathrm{H}, H_{\mathrm{m}}\right) 4.42\left(\mathrm{~m}, 1 \mathrm{H}, H_{2}\right), 4.93\left(\mathrm{~m}, 4 \mathrm{H}, H_{\mathrm{a}}\right), 5.10(\mathrm{~s}, 2 \mathrm{H}$, $\left.H_{6}\right), 5.77\left(\mathrm{~m}, 2 \mathrm{H}, H_{\mathrm{b}}\right), 6.43\left(\mathrm{~d},{ }^{3} J_{\mathrm{H}, \mathrm{H}}=7.2 \mathrm{~Hz}, 1 \mathrm{H}, H_{13}\right), 7.31-7.45$ (m, $\left.5 \mathrm{H}, H_{8}-H_{12}\right)$.

$N$ - + C-Glu (2a): ${ }^{13} \mathrm{C}-\mathrm{NMR}\left(\mathrm{CDCl}_{3}, 27{ }^{\circ} \mathrm{C}, 100 \mathrm{MHz}\right): \delta[\mathrm{ppm}]$ $25.5\left(C_{\mathrm{i}}\right), 26.8\left(C_{\mathrm{i}}\right), 27.8\left(C_{3}\right), 28.9-29.5\left(C_{\mathrm{d}}-C_{\mathrm{h}}+C_{1}\right), 30.6\left(C_{4}\right)$, $33.8\left(C_{\mathrm{c}}\right), 36.5\left(C_{\mathrm{j}}\right), 39.6\left(C_{\mathrm{m}}\right), 52.3\left(C_{2}\right), 66.6\left(C_{6}\right), 114.1\left(C_{\mathrm{a}}\right)$, 128.2-128.7 $\left(C_{8}-C_{12}\right), 135.8\left(C_{7}\right), 139.1\left(C_{\mathrm{b}}\right), 170.9\left(C_{1}\right), 173.4$ $\left(C_{5}\right), 173.4\left(C_{\mathrm{k}}\right)$.

$N$ - $+C$-Asp (2b): ${ }^{1} \mathrm{H}-\mathrm{NMR}\left(\mathrm{CDCl}_{3}, 27{ }^{\circ} \mathrm{C}, 400 \mathrm{MHz}\right): \delta[\mathrm{ppm}]$ 1.27-1.40 (m, 22H, $\left.H_{\mathrm{d}}-H_{\mathrm{h}}+H_{\mathrm{l}}\right), 1.59\left(\mathrm{~m}, 2 \mathrm{H}, H_{\mathrm{i}}\right), 2.02-2.20(\mathrm{~m}$, $\left.6 \mathrm{H}, H_{4}+H_{\mathrm{c}}+H_{\mathrm{j}}\right), 2.91\left(\mathrm{~m}, 1 \mathrm{H}, H_{3}\right), 3.08\left(\mathrm{~m}, 1 \mathrm{H}, H_{3}\right), 3.17(\mathrm{~m}, 2 \mathrm{H}$, $\left.H_{\mathrm{m}}\right), 4.91\left(\mathrm{~m} ; 1 \mathrm{H}, H_{2}\right), 4.94\left(\mathrm{~m}, 2 \mathrm{H}, H_{\mathrm{a}}\right), 5.14\left(\mathrm{~s}, 2 \mathrm{H}, H_{5}\right), 5.79(\mathrm{~m}$, $\left.1 \mathrm{H}, H_{\mathrm{b}}\right), 6.56\left(\mathrm{~d},{ }^{3} J_{\mathrm{H}, \mathrm{H}}=7.2 \mathrm{~Hz}, 1 \mathrm{H}, H_{12}\right), 7.31-7.45\left(\mathrm{~m}, 5 \mathrm{H}, H_{7}-\right.$ $\left.H_{11}\right)$.

$N-+C$-Asp (2b): ${ }^{13} \mathrm{C}-\mathrm{NMR}\left(\mathrm{CDCl}_{3}, 27{ }^{\circ} \mathrm{C}, 100 \mathrm{MHz}\right): \delta[\mathrm{ppm}]$ $25.5\left(C_{\mathrm{i}}\right), 26.8\left(C_{\mathrm{i}}\right), 28.9-29.3\left(C_{\mathrm{d}}-C_{\mathrm{h}}+C_{\mathrm{l}}\right), 33.7\left(C_{\mathrm{c}}\right), 35.7\left(C_{\mathrm{j}}\right)$, $36.5\left(C_{3}\right), 39.6\left(C_{\mathrm{m}}\right), 49.1\left(C_{2}\right), 66.9\left(C_{5}\right), 114.1\left(C_{\mathrm{a}}\right), 128.2-128.6$ $\left(C_{7}-C_{11}\right), 135.4\left(C_{6}\right), 139.2\left(C_{\mathrm{b}}\right), 170.1\left(C_{1}\right), 172.1\left(C_{4}\right), 173.3\left(C_{\mathrm{k}}\right)$.

$N$ - $+C$-Leu (2c): ${ }^{1} \mathrm{H}-\mathrm{NMR}\left(\mathrm{CDCl}_{3}, 27{ }^{\circ} \mathrm{C}, 400 \mathrm{MHz}\right): \delta[\mathrm{ppm}]$ $0.95\left(\mathrm{~m}, 6 \mathrm{H}, H_{5}+H_{6}\right), 1.28-1.43\left(\mathrm{~m}, 22 \mathrm{H}, H_{\mathrm{d}}-H_{\mathrm{h}}+H_{\mathrm{l}}\right), 1.60-$ $1.71\left(\mathrm{~m}, 5 \mathrm{H}, H_{\mathrm{i}}+H_{3}+H_{4}\right), 2.02\left(\mathrm{~m}, 2 \mathrm{H}, H_{\mathrm{c}}\right), 2.20\left(\mathrm{~m}, 2 \mathrm{H}, H_{\mathrm{j}}\right), 3.22$ $\left(\mathrm{m}, 2 \mathrm{H}, H_{\mathrm{m}}\right), 4.61\left(\mathrm{~m}, 1 \mathrm{H}, H_{2}\right), 4.94\left(\mathrm{~m}, 2 \mathrm{H}, H_{\mathrm{a}}\right), 5.79\left(\mathrm{~m}, 1 \mathrm{H}, H_{\mathrm{b}}\right)$, $5.96\left(\mathrm{~m}, 1 \mathrm{H}, H_{7}\right)$.

$N-+C$-Leu (2c): ${ }^{13} \mathrm{C}-\mathrm{NMR}\left(\mathrm{CDCl}_{3}, 27{ }^{\circ} \mathrm{C}, 100 \mathrm{MHz}\right): \delta[\mathrm{ppm}]$ 21.9-22.6 $\left(C_{5}+C_{6}\right), 25.0\left(C_{4}\right), 25.6\left(C_{\mathrm{i}}\right), 26.8\left(C_{\mathrm{i}}\right), 28.9-29.5\left(C_{\mathrm{d}}-\right.$ $\left.C_{\mathrm{h}}\right), 33.7\left(C_{\mathrm{c}}\right), 36.6\left(C_{\mathrm{j}}\right), 39.5\left(C_{\mathrm{m}}\right), 41.1\left(C_{3}\right), 51.6\left(C_{2}\right), 114.1\left(C_{\mathrm{a}}\right)$, $139.1\left(C_{\mathrm{b}}\right), 172.0\left(C_{1}\right), 173.1\left(C_{\mathrm{k}}\right)$.

General procedure for achiral AA (2d-2e). Reactions were performed under $\mathrm{N}_{2}$ atmosphere. To a solution of $\mathrm{N}$-terminus functionalized amino acids 1d-1e (1.0 eq.) and $\mathbf{A 2}$ (1.0 eq.) in $0{ }^{\circ} \mathrm{C}$ cold dried dichloromethane $(20 \mathrm{~mL})$ DIPEA (4.5 eq.), DMAP (0.02 eq.) and EDC $\cdot \mathrm{HCl}$ (2.6 eq.) were added successively. The solution was heated to reflux for $24 \mathrm{~h}$ followed by extraction with brine $(20 \mathrm{~mL}), 1 \mathrm{M} \mathrm{HCl}(20 \mathrm{~mL})$, brine $(20 \mathrm{~mL})$, saturated $\mathrm{NaHCO}_{3}$-solution $(20 \mathrm{~mL})$ and brine $(20 \mathrm{~mL})$. The organic layer was separated, dried over $\mathrm{Na}_{2} \mathrm{SO}_{4}$ and concentrated in vacuum to yield $2 \mathbf{d}-2 \mathrm{e}(78-82 \%)$.

$N$ - $+C$-Aib (2d): ${ }^{1} \mathrm{H}-\mathrm{NMR}\left(\mathrm{CDCl}_{3}, 27{ }^{\circ} \mathrm{C}, 400 \mathrm{MHz}\right): \delta[\mathrm{ppm}]$ 1.27-1.36 (m, 20H, $\left.H_{\mathrm{d}}-H_{\mathrm{h}}\right), 1.50\left(\mathrm{~m}, 2 \mathrm{H}, H_{\mathrm{l}}\right), 1.55-1.62(\mathrm{~m}, 8 \mathrm{H}$, $\left.H_{2}+H_{3}+H_{\mathrm{i}}\right), 2.04\left(\mathrm{~m}, 4 \mathrm{H}, H_{\mathrm{c}}\right), 2.16\left(\mathrm{~m}, 2 \mathrm{H}, H_{\mathrm{j}}\right), 3.23(\mathrm{~m}, 2 \mathrm{H}$, $\left.H_{\mathrm{m}}\right), 4.96\left(\mathrm{~m}, 2 \mathrm{H}, H_{\mathrm{a}}\right), 5.81\left(\mathrm{~m}, 1 \mathrm{H}, H_{\mathrm{b}}\right), 6.08\left(\mathrm{~m}, 1 \mathrm{H}, H_{5}\right), 6.65$ (m, $\left.1 \mathrm{H}, H_{5}\right)$.

$N-+C$-Aib (2d): ${ }^{13} \mathrm{C}-\mathrm{NMR}\left(\mathrm{CDCl}_{3}, 27{ }^{\circ} \mathrm{C}, 100 \mathrm{MHz}\right): \delta[\mathrm{ppm}]$ $24.8\left(C_{2}+C_{3}\right), 25.5\left(C_{\mathrm{i}}\right), 26.8\left(C_{\mathrm{i}}\right), 28.9-29.5\left(C_{\mathrm{d}}-C_{\mathrm{h}}\right), 33.8\left(C_{\mathrm{c}}\right)$,
$37.4\left(C_{\mathrm{j}}\right), 39.8\left(C_{\mathrm{j}}\right), 57.4\left(C_{1}\right), 114.1\left(C_{\mathrm{a}}\right), 139.1\left(C_{\mathrm{b}}\right), 173.4\left(C_{\mathrm{k}}\right)$, $174.5\left(C_{4}\right)$.

$N$ - $+C$-ACHC (2e): ${ }^{1} \mathrm{H}-\mathrm{NMR}\left(\mathrm{CDCl}_{3}, 27{ }^{\circ} \mathrm{C}, 400 \mathrm{MHz}\right): \delta[\mathrm{ppm}]$ 1.29-1.47 (m, 24H, $\left.H_{\mathrm{d}}-H_{\mathrm{i}}+H_{\mathrm{l}}\right), 1.64\left(\mathrm{~m}, 6 \mathrm{H}, H_{3}+H_{4}+H_{5}\right), 1.88$ $\left(\mathrm{m}, 2 \mathrm{H}, H_{2}+H_{6}\right), 2.04\left(\mathrm{~m}, 6 \mathrm{H}, H_{\mathrm{c}}+H_{2}+H_{6}\right), 2.21\left(\mathrm{~m}, 2 \mathrm{H}, H_{\mathrm{j}}\right), 3.20$ $\left(\mathrm{m}, 2 \mathrm{H}, H_{\mathrm{m}}\right), 4.97\left(\mathrm{~m}, 4 \mathrm{H}, H_{\mathrm{a}}\right), 5.30\left(\mathrm{~m}, 1 \mathrm{H}, H_{7}\right), 5.79\left(\mathrm{~m}, 2 \mathrm{H}, H_{\mathrm{b}}\right)$.

$N$ - $+C$-ACHC (2e): ${ }^{13} \mathrm{C}-\mathrm{NMR}\left(\mathrm{CDCl}_{3}, 27{ }^{\circ} \mathrm{C}, 100 \mathrm{MHz}\right): \delta[\mathrm{ppm}]$ $21.6\left(C_{3}+C_{5}\right), 25.1-25.5\left(C_{\mathrm{i}}+C_{4}\right), 26.9\left(C_{\mathrm{i}}\right), 28.9-29.5\left(C_{\mathrm{d}}-C_{\mathrm{h}}+\right.$ $\left.C_{\mathrm{l}}\right), 32.0-33.8\left(C_{2}+C_{6}+C_{\mathrm{c}}\right), 37.5\left(C_{\mathrm{j}}\right), 39.5\left(C_{\mathrm{m}}\right), 59.7\left(C_{1}\right), 114.1$ $\left(C_{\mathrm{a}}\right), 139.1\left(C_{\mathrm{b}}\right), 175.1\left(C_{\mathrm{k}}\right), 176.0\left(C_{4}\right)$.

ADMET-polymerization. Polymerization of the monomers 2a-2e was done as bulk polymerization, following the methods proposed by Wagener et al. in the past. ${ }^{16}$ The solid monomers were placed in a dried Schlenk tube, removing oxygen by flushing with nitrogen, subsequently heating in an oil bath to $65{ }^{\circ} \mathrm{C}$. After the monomer becomes liquid, the appropriate amount of the chosen catalyst (100:1 monomer to catalyst ratio) was added to the bulk. Vacuum and repeated cycles flushing with nitrogen are applied during the reaction to remove evolving ethylene, further small amount of catalyst were additionally added to the bulk-reaction mixture after several hours. Due to increasing viscosity during polymerization the reaction temperature was increased stepwise starting from $65^{\circ} \mathrm{C}$ up to $165^{\circ} \mathrm{C}$ (for the last hour) and afterwards the reaction was quenched by adding THF and precipitating of the obtained polymer into cold $\mathrm{MeOH}$ to yield 3a-3e (61-87\%).

ADMET-Glu (3a): ${ }^{1} \mathrm{H}-\mathrm{NMR}\left(\mathrm{CDCl}_{3}, 27{ }^{\circ} \mathrm{C}, 400 \mathrm{MHz}\right): \delta[\mathrm{ppm}]$ 1.26-1.34 (m, $\left.\mathrm{H}_{\text {rep.unit }}, H_{\mathrm{d}}-H_{\mathrm{h}}\right), 1.44-1.59$ (m, $\mathrm{H}_{\text {rep.unit }}, H_{\mathrm{i}}+H_{\mathrm{l}}$ ), 1.95-2.01 (m, $\left.\mathrm{H}_{\text {rep.unit }}, H_{4}+H_{\mathrm{c}}\right), 2.16$ (m, $\left.\mathrm{H}_{\text {rep.unit }}, H_{\mathrm{j}}\right), 2.41$ (m, $\left.\mathrm{H}_{\text {rep.unit }}, H_{3}\right), 2.55\left(\mathrm{~m}, \mathrm{H}_{\text {rep.unit }}, H_{3}\right), 3.20\left(\mathrm{~m}, \mathrm{H}_{\text {rep.unit }}, H_{\mathrm{m}}\right) 4.41(\mathrm{~m}$, $\left.\mathrm{H}_{\text {rep.unit }}, H_{2}\right), 4.96\left(\mathrm{~m}, 4 \mathrm{H}, H_{\mathrm{a}}\right), 5.10$ (s, $\left.\mathrm{H}_{\text {rep.unit }}, H_{6}\right), 5.36$ (m, $\left.\mathrm{H}_{\text {rep.unit }}, H_{x}+H_{y}\right), 5.79\left(\mathrm{~m}, 2 \mathrm{H}, H_{\mathrm{b}}\right), 7.34\left(\mathrm{~m}, \mathrm{H}_{\text {rep.unit }}, H_{8}-H_{12}\right)$. ADMET-Asp (3b): ${ }^{1} \mathrm{H}-\mathrm{NMR}\left(\mathrm{CDCl}_{3}, 27{ }^{\circ} \mathrm{C}, 400 \mathrm{MHz}\right): \delta[\mathrm{ppm}]$ 1.19-1.28 (m, $\left.\mathrm{H}_{\text {rep.Einheit }}, H_{\mathrm{d}}-H_{\mathrm{h}}\right), 1.43\left(\mathrm{~m}, \mathrm{H}_{\text {rep.Einheit }}, H_{\mathrm{l}}\right), 1.58$ $\left(\mathrm{m}, \mathrm{H}_{\text {rep.Einheit }}, H_{\mathrm{i}}\right), 1.95\left(\mathrm{~m}, \mathrm{H}_{\text {rep.Einheit }}, H_{\mathrm{c}}\right), 2.18\left(\mathrm{~m}, \mathrm{H}_{\text {rep.Einheit }}\right.$, $\left.H_{\mathrm{j}}\right), 2.68\left(\mathrm{~m}, \mathrm{H}_{\text {rep.Einheit }}, H_{3}\right), 2.90\left(\mathrm{~m}, \mathrm{H}_{\text {rep.Einheit }}, H_{3}\right), 3.16$ $\left(\mathrm{m}, \mathrm{H}_{\text {rep.Einheit }}, H_{\mathrm{m}}\right), 4.78\left(\mathrm{~m}, \mathrm{H}_{\text {rep.Einheit }}, H_{2}\right), 4.96\left(\mathrm{~m}, 4 \mathrm{H}, H_{\mathrm{a}}\right)$, $5.13\left(\mathrm{~m}, \mathrm{H}_{\text {rep.Einheit }}, H_{5}\right), 5.36\left(\mathrm{~m}, \mathrm{H}_{\text {rep.Einheit }}, H_{x}+H_{y}\right), 5.79$ (m, 2H, $\left.H_{\mathrm{b}}\right), 7.33\left(\mathrm{~m}, \mathrm{H}_{\text {rep.Einheit }}, H_{7}-H_{11}\right)$.

ADMET-Leu (3c): ${ }^{1} \mathrm{H}-\mathrm{NMR}\left(\mathrm{CDCl}_{3}, 27{ }^{\circ} \mathrm{C}, 400 \mathrm{MHz}\right): \delta[\mathrm{ppm}]$ 0.87 (m, $\left.\mathrm{H}_{\text {rep.Einheit }}, H_{5}+H_{6}\right), 1.19-1.38\left(\mathrm{~m}, 12 \mathrm{H}, H_{\mathrm{d}}-H_{\mathrm{h}}+H_{1}\right)$, 1.46-1.66 (m, $\left.\mathrm{H}_{\text {rep.Einheit }}, H_{3}+H_{4}+H_{\mathrm{i}}\right), 2.03\left(\mathrm{~m}, \mathrm{H}_{\text {rep.Einheit }}, H_{\mathrm{c}}\right)$, $2.17\left(\mathrm{~m}, \mathrm{H}_{\text {rep.Einheit }}, H_{\mathrm{j}}\right), 3.19\left(\mathrm{~m}, \mathrm{H}_{\text {rep.Einheit, }} H_{\mathrm{l}}\right), 4.48(\mathrm{~m}$, $\left.\mathrm{H}_{\text {rep.Einheit }}, H_{2}\right), 4.90\left(\mathrm{~m}, 4 \mathrm{H}, H_{\mathrm{a}}\right), 5.35\left(\mathrm{~m}, \mathrm{H}_{\text {rep.Einheit }}, H_{x}+H_{y}\right)$, $5.78\left(\mathrm{~m}, 2 \mathrm{H}, H_{\mathrm{b}}\right)$.

ADMET-Aib (3d): ${ }^{1} \mathrm{H}-\mathrm{NMR}\left(\mathrm{CDCl}_{3}, 27{ }^{\circ} \mathrm{C}, 400 \mathrm{MHz}\right): \delta[\mathrm{ppm}]$ $1.27-1.36\left(\mathrm{~m}, \mathrm{H}_{\text {rep.unit }}, H_{\mathrm{d}}-H_{\mathrm{h}}\right), 1.48\left(\mathrm{~m}, \mathrm{H}_{\text {rep.unit }}, H_{1}\right), 1.55-1.59$ $\left(\mathrm{m}, \mathrm{H}_{\text {rep.unit }}, H_{2}+H_{3}+H_{\mathrm{i}}\right), 1.94\left(\mathrm{~m}, \mathrm{H}_{\text {rep.unit }}, H_{\mathrm{c}}\right), 2.16$ (m, $\left.\mathrm{H}_{\text {rep.unit }}, H_{\mathrm{j}}\right), 3.23$ (m, $\left.\mathrm{H}_{\text {rep.unit }}, H_{\mathrm{m}}\right), 4.96\left(\mathrm{~m}, 2 \mathrm{H}, H_{\mathrm{a}}\right), 5.36$ $\left(\mathrm{m}, \mathrm{H}_{\text {rep.unit }}, H_{x}+H_{y}\right), 5.76\left(\mathrm{~m}, 1 \mathrm{H}, H_{\mathrm{b}}\right), 6.08\left(\mathrm{~m}, \mathrm{H}_{\text {rep.unit }}, H_{5}\right)$, $6.65\left(\mathrm{~m}, \mathrm{H}_{\text {rep.unit }}, H_{6}\right)$.

ADMET-ACHC (3e): ${ }^{1} \mathrm{H}-\mathrm{NMR}\left(\mathrm{CDCl}_{3}, 27{ }^{\circ} \mathrm{C}, 400 \mathrm{MHz}\right)$ : $\delta[\mathrm{ppm}]$ 1.23-1.47 (m, $\left.\mathrm{H}_{\text {rep.unit }}, H_{\mathrm{d}}-H_{\mathrm{h}}+H_{1}+H_{\mathrm{i}}\right), 1.62$ (m, $\left.\mathrm{H}_{\text {rep.unit }}, H_{3}+H_{4}+H_{5}\right), 1.86\left(\mathrm{~m}, \mathrm{H}_{\text {rep.unit }}, H_{2}+H_{6}\right), 1.94-2.12$ $\left(\mathrm{m}, \mathrm{H}_{\text {rep.unit }}, H_{\mathrm{c}}+H_{2}+H_{6}\right), 2.21\left(\mathrm{~m}, \mathrm{H}_{\text {rep.unit }}, H_{\mathrm{j}}\right), 3.20$ $\left(\mathrm{m}, \mathrm{H}_{\text {rep.unit }}, H_{\mathrm{m}}\right), 4.91\left(\mathrm{~m}, 4 \mathrm{H}, H_{\mathrm{a}}\right), 5.35\left(\mathrm{~m}, \mathrm{H}_{\text {rep.unit }}, H_{x}+\mathrm{H}_{\mathrm{y}}\right)$, $5.78\left(\mathrm{~m}, 2 \mathrm{H}, H_{\mathrm{b}}\right)$. 
Hydrogenation/deprotection of the polymers

General procedure for hydrogenation and deprotection of the polymers bearing benzyl-protecting group in the side chain $(\mathbf{4 a}-\mathbf{4 b})$. To a solution of the polymer 3a-3b (1.0 eq.) in DMF $\mathrm{TsNHNH}_{2}$ (5.0 eq. per double bond) and DIPEA (5.0 eq.) were added. The solution was heated up to $150{ }^{\circ} \mathrm{C}$ for 6 hours. Then the solution was cooled to $85{ }^{\circ} \mathrm{C}, \mathrm{NaOH}$ (2.0 eq. per protection group) was added and the resulting mixture was stirred overnight. The reaction mixture was cooled to room temperature and dialyzed against $\mathrm{MeOH}$ for 3 days to yield the hydrogenated and deprotected polymers $\mathbf{4 a}-\mathbf{4 b}(\mathbf{7 2 - 7 9 \% )}$.

ADMET-Glu H (4a): ${ }^{1} \mathrm{H}-\mathrm{NMR}\left(\mathrm{CDCl}_{3}+15\right.$ vol\% TFA, $27{ }^{\circ} \mathrm{C}$, $400 \mathrm{MHz}): \delta[\mathrm{ppm}] 0.87\left(\mathrm{~m}, 6 \mathrm{H}, H_{z}\right), 1.25-1.34\left(\mathrm{~m}, \mathrm{H}_{\text {rep.unit }}, H_{\mathrm{b}}-\right.$ $\left.H_{\mathrm{h}}+H_{x}+H_{y}\right), 1.54-1.60\left(\mathrm{~m}, \mathrm{H}_{\text {rep.unit }}, H_{\mathrm{i}}+H_{\mathrm{l}}\right), 2.00-2.16$ (m, $\left.\mathrm{H}_{\text {rep.unit }}, H_{3}\right), 2.39$ (m, $\left.\mathrm{H}_{\text {rep.unit }}, H_{4}\right), 2.54$ (m, $\mathrm{H}_{\text {rep.unit }}, H_{\mathrm{j}}$ ), 3.29 (m, $\left.\mathrm{H}_{\text {rep.unit }}, H_{\mathrm{m}}\right), 4.73$ (m, $\left.\mathrm{H}_{\text {rep.unit }}, H_{2}\right), 7.21$ (m, $\mathrm{H}_{\text {rep.unit }}$, $\left.H_{13}\right), 7.81$ (m, $\left.\mathrm{H}_{\text {rep.unit }}, H_{14}\right)$.

ADMET-Asp H (4b): ${ }^{1} \mathrm{H}-\mathrm{NMR}\left(\mathrm{CDCl}_{3}+15\right.$ vol\% TFA, $27{ }^{\circ} \mathrm{C}, 400$ $\mathrm{MHz}): \delta[\mathrm{ppm}] 0.87\left(\mathrm{~m}, 6 \mathrm{H}, H_{z}\right), 1.19-1.28\left(\mathrm{~m}, \mathrm{H}_{\text {rep.unit }}, H_{\mathrm{b}}-H_{\mathrm{h}}+\right.$ $\left.H_{x}+H_{y}\right), 1.59\left(\mathrm{~m}, \mathrm{H}_{\text {rep.Einheit }}, H_{\mathrm{i}}+H_{\mathrm{l}}\right), 2.35\left(\mathrm{~m}, \mathrm{H}_{\text {rep.Einheit }}, H_{\mathrm{j}}\right), 3.01$ (m, $\left.\mathrm{H}_{\text {rep.Einheit }}, H_{3}\right), 3.24$ (m, $\left.\mathrm{H}_{\text {rep.Einheit }}, H_{3}\right), 3.59\left(\mathrm{~m}, \mathrm{H}_{\text {rep.Einheit }}\right.$, $\left.H_{\mathrm{m}}\right), 4.50$ (m, $\left.\mathrm{H}_{\text {rep.Einheit }}, H_{2}\right), 7.05$ (m, $\left.\mathrm{H}_{\text {rep.unit }}, H_{12}+H_{13}\right)$.

General procedure for hydrogenation of the polymers without a protecting group in the side chain $(\mathbf{4 c - 4 e )}$. To a solution of the polymer 3c-3e (1.0 eq.) in $\mathrm{DMF}^{\mathrm{TsNHNH}} \mathrm{H}_{2}$ (5.0 eq. per double bond) and DIPEA (5.0 eq.) were added. The solution was heated up to $150{ }^{\circ} \mathrm{C}$ for 6 hours. The resulting mixture was cooled to room temperature and dialyzed against $\mathrm{MeOH}$ for 3 days to yield the hydrogenated polymers 4c-4e (67-83\%).

ADMET-Leu H (4c): ${ }^{1} \mathrm{H}-\mathrm{NMR}\left(\mathrm{CDCl}_{3}+15\right.$ vol\% TFA, $27{ }^{\circ} \mathrm{C}, 400$ MHz): $\delta[\mathrm{ppm}] 0.89\left(\mathrm{~m}, \mathrm{H}_{\text {rep.Einheit }}, H_{5}+H_{6}+H_{z}\right), 1.19-1.38(\mathrm{~m}$, $\left.12 \mathrm{H}, H_{\mathrm{b}}-H_{\mathrm{h}}+H_{x}+H_{y}\right), 1.50-1.59\left(\mathrm{~m}, \mathrm{H}_{\text {rep.Einheit }}, H_{3}+H_{4}+H_{\mathrm{i}}+\right.$ $\left.H_{\mathrm{l}}\right), 2.32\left(\mathrm{~m}, \mathrm{H}_{\text {rep.Einheit }}, H_{\mathrm{j}}\right), 3.25\left(\mathrm{~m}, \mathrm{H}_{\text {rep.Einheit }}, H_{\mathrm{m}}\right), 4.54(\mathrm{~m}$, $\left.\mathrm{H}_{\text {rep.Einheit }}, H_{2}\right), 7.19$ (m, $\left.\mathrm{H}_{\text {rep.Einheit }}, H_{7}\right), 7.39$ (m, $\left.\mathrm{H}_{\text {rep.Einheit }}, H_{8}\right)$.

ADMET-Aib H (4d): ${ }^{1} \mathrm{H}-\mathrm{NMR}\left(\mathrm{CDCl}_{3}+15\right.$ vol\% TFA, $27{ }^{\circ} \mathrm{C}$, $400 \mathrm{MHz}): \delta[\mathrm{ppm}] 0.88\left(\mathrm{~m}, 6 \mathrm{H}, H_{z}\right), 1.25\left(\mathrm{~m}, \mathrm{H}_{\text {rep.unit }}, H_{\mathrm{b}}-H_{\mathrm{h}}+\right.$ $\left.H_{1}+H_{x}+H_{y}\right), 1.55-1.59\left(\mathrm{~m}, \mathrm{H}_{\text {rep.unit }}, H_{2}+H_{3}+H_{\mathrm{i}}\right), 2.28$ (m, $\left.\mathrm{H}_{\text {rep.unit }}, H_{\mathrm{j}}\right), 3.29$ (m, $\left.\mathrm{H}_{\text {rep.unit }}, H_{\mathrm{m}}\right), 6.70$ (m, $\mathrm{H}_{\text {rep.unit }}, H_{5}$ ), $6.99\left(\mathrm{~m}, \mathrm{H}_{\text {rep.unit }}, H_{6}\right)$.

ADMET-ACHC H (4e): ${ }^{1} \mathrm{H}-\mathrm{NMR}\left(\mathrm{CDCl}_{3}+15\right.$ vol\% TFA, $27{ }^{\circ} \mathrm{C}$, $400 \mathrm{MHz}): \delta[\mathrm{ppm}] 0.88\left(\mathrm{~m}, 6 \mathrm{H}, H_{z}\right), 1.27-1.46\left(\mathrm{~m}, \mathrm{H}_{\text {rep.unit }}, H_{\mathrm{b}}-\right.$ $\left.H_{\mathrm{h}}+H_{1}+H_{x}+H_{y}+H_{\mathrm{i}}\right), 1.74\left(\mathrm{~m}, \mathrm{H}_{\text {rep.unit }}, H_{3}+H_{4}+H_{5}\right), 1.96-2.31$ (m, $\left.\mathrm{H}_{\text {rep.unit }}, H_{\mathrm{j}}+H_{2}+H_{6}\right), 3.37\left(\mathrm{~m}, \mathrm{H}_{\text {rep.unit }}, H_{\mathrm{m}}\right), 7.12-7.26$ (m, $\mathrm{H}_{\text {rep.unit }}, H_{7}+\mathrm{H}_{8}$ ).

\section{Results and discussion}

The synthesis of the polymers was accomplished by ADMET polymerization of the chosen $N$ - and $C$-terminus $\omega$-alkene-chain functionalized amino acids. For the investigation of the influence on the chiral/achiral and polar/nonpolar nature of the amino acids on the crystallization behavior, chiral/polar amino acids glutamic (Glu) and aspartic acid (Asp), chiral/unpolar L-leucine (Leu) and achiral/unpolar 2-amino-2-methylpropanoic (Aib) acid and 1-aminocyclohexane-carboxylic acid (ACHC) were functionalized with terminal $\omega$-alkenyl-group, polymerized by ADMET and hydrogenated (Fig. 1).

\section{Monomer synthesis}

Conceptually, the amino acids were introduced in the polymer structure by the fixation of two $\omega$-alkenyl-chains of equal length on either side. Therefore, $N$ - and $C$-terminus functionalization was realized by reaction of the amino acid with 10-undecenoyl chloride and afterwards coupling with 10-undecen-1-amine (A2) (see Scheme 1).

The chosen method and the yields of the functionalization reactions for the different amino acids are shown in Table 1.

For $N$-terminus functionalization pyridine was used for the protected L-glutamic acid and L-aspartic acid $\mathbf{1 a - 1 b}$ or alternatively via a two phase system of aqueous $\mathrm{NaOH} / \mathrm{CH}_{2} \mathrm{Cl}_{2}$ mixture (Schotten-Baumann-method) for the synthesis of 1c-1e. Free carboxylic group in products 1a-1e could then be reacted with 10undecen-1-amine (A2), which was synthesized before in a two-step synthesis by reaction of 10-undecenoyl chloride with ammoniumhydroxid to the corresponding amide (A1), followed by reduction with $\mathrm{LiAlH}_{4}$ as described in the literature. ${ }^{45}$ All synthesized monomers 1a-1e and 2a-2b were analysed by ${ }^{1} \mathrm{H},{ }^{13} \mathrm{C}-\mathrm{NMR}$ and ESI-ToF-MS spectroscopy (see Fig. S1-S28 ESI $\dagger$ ), A1-A2 were analysed by ${ }^{1} \mathrm{H}$ and ${ }^{13} \mathrm{C}-\mathrm{NMR}$ spectroscopy (see Fig. S29-S30 ESI $\dagger$ ).

\section{ADMET-polymerization and hydrogenation of the polymers}

Synthesis of the precision PE-polymers was realized by ADMETpolymerization followed by hydrogenation with $p$-toulenesulfonhydrazide $\left(\mathrm{TsNHNH}_{2}\right)$ as shown in Scheme 1.

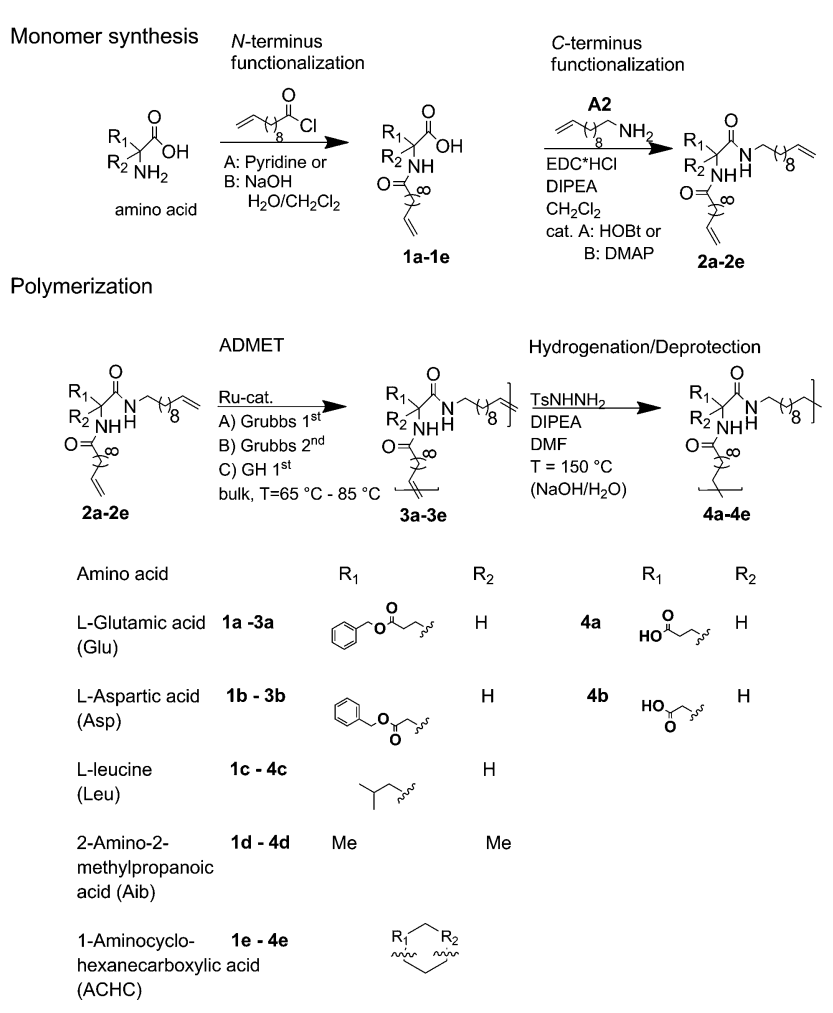

Scheme 1 Synthesis for (a) $N$ - + C-terminus functionalized monomers $2 \mathrm{a}-2 \mathrm{e}$ and (b) ADMET-polymers $3 \mathrm{a}-3 \mathrm{e}$ and hydrogenated/ deprotected polymers $4 a-4 e$. 
Table 1 Chosen methods and yields for $\mathrm{N}$ - and $\mathrm{C}$-terminus functionalization of the different amino acids

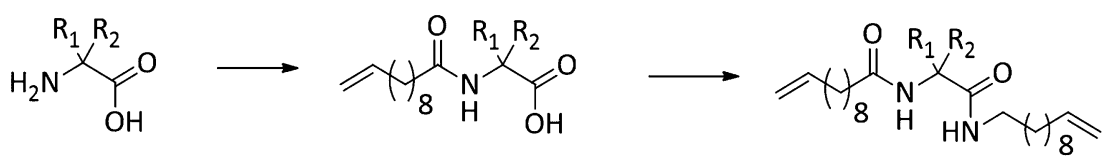

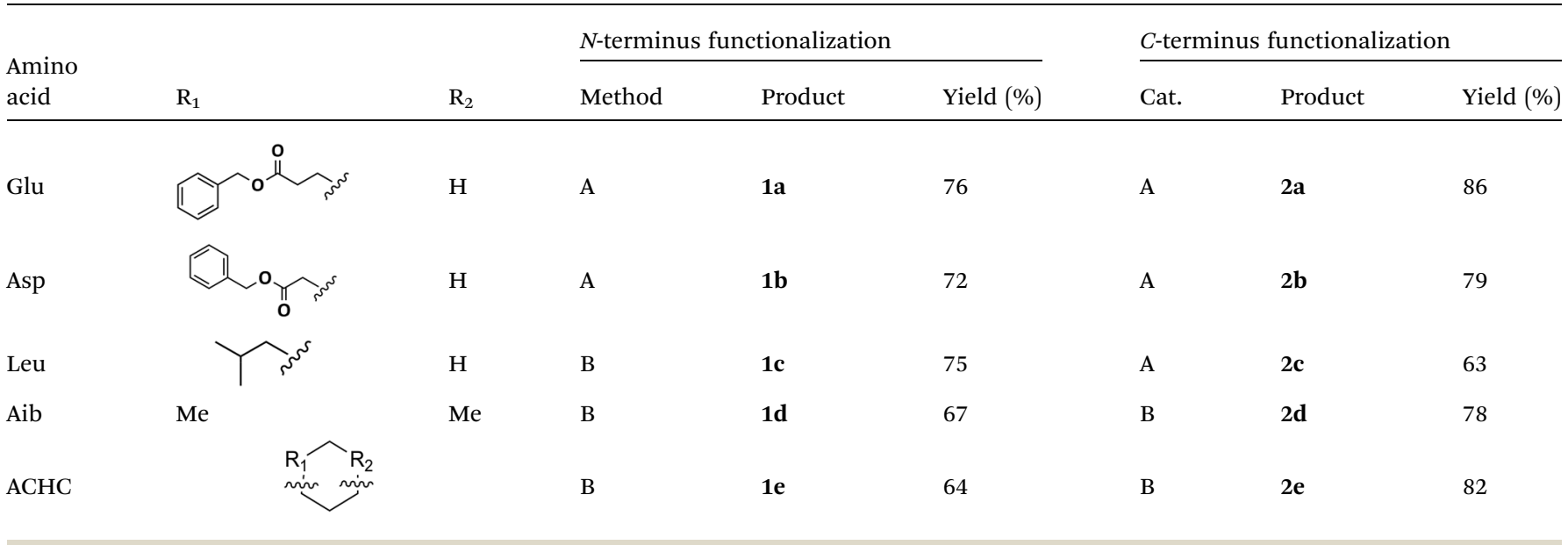

Polymerization of the monomers $2 \mathbf{a}-\mathbf{2 e}$ can be done as a bulk polymerization due to the low melting points. The solid monomers were heated up in an oil bath to $65{ }^{\circ} \mathrm{C}$ till they became completely liquid. ${ }^{16}$ The appropriate amount of catalyst (100 : 1 monomer to catalyst ratio) was added to the monomermelt in which the same amount of catalyst was additionally added to the bulk after several hours. Due to the increasing viscosity during polymerization the reaction temperature was increased stepwise from $85^{\circ} \mathrm{C}$ up to $165^{\circ} \mathrm{C}$. Finally the reaction was quenched by adding THF and precipitating the obtained polymer into cold $\mathrm{MeOH}$.

Due to different activity and isomerization rates ${ }^{46-49}$ Grubbs Catalyst first generation (Grubbs $1^{\text {st }}$ ), second generation (Grubbs $2^{\text {nd }}$ ) and Hoveyda-Grubbs Catalyst first generation (GH $\left.1^{\text {st }}\right)$ were tested for the chosen reaction conditions. In Table 2 the results of the molecular weights (obtained by NMR and GPC as well as the PDI's), the yields and the obtained trans : cis ratio of the internal double bonds for the different catalyst are displayed. A low isomerization rate ${ }^{48}$ and good yields for monomers $3 \mathbf{a}-3 \mathbf{c}$ by using Grubbs $1^{\text {st }}$ catalyst were obtained. GH $1^{\text {st }}$ catalyst under complete oxygen-free conditions also achieved good results with respect to yield and molecular weights of the products, whereas Grubbs $2^{\text {nd }}$ catalyst was found to be the catalyst resulting in the lowest molecular weights. Probing the optimized conditions described by Wagener et al. ${ }^{16}$ molecular weights as high as $22 \mathrm{kDa}$ were obtained - higher molecular weights were not obtained. This might be due to the high temperature at which most of the monomers are melting (often above $165{ }^{\circ} \mathrm{C}$ ), which is limiting the activity of the catalysts. Even though such high reaction temperatures could lead to various side reactions, only a low isomerization rate was observed in MALDI-Tof-MS analysis.

Hydrogenation of the terminal and internal double bonds in the backbone to yield the polymer $\mathbf{4 a - 4 e}$ was realized by reaction of the polymers with $p$-toluenesulfonhydrazide
$\left(\mathrm{TsNHNH}_{2}\right)$ and DIPEA in DMF at $150{ }^{\circ} \mathrm{C}$ according to literature $^{50-53}$ (see Scheme 1). For the hydrogenation the ADMET polymers $3 a-3 e$ synthesized with Grubbs $1^{\text {st }}$ generation catalyst were used and molecular weights from $3100 \mathrm{~g} \mathrm{~mol}^{-1}$ to $18500 \mathrm{~g} \mathrm{~mol}^{-1}$ were obtained (Table 3). Reactions were stopped after $6 \mathrm{~h}$, complete deprotection of the asparagine and glutamic acid unit in the polymer backbone could be realized by subsequent ester hydrolysis with aqueous $\mathrm{NaOH}$ solution. The resulting mixture was dialysed against $\mathrm{MeOH}$ for 3 days to remove the obtained $p$-toluenesulfonic acid and benzyl alcohol in case of $\mathbf{4 a}$ and $\mathbf{4 b}$. During dialysis, the obtained hydrogenated polymers precipitated due to their complete insolubility in the organic solvents used. The monomer and polymer synthesis as well as hydrogenation/ deprotection can be followed by ${ }^{1} \mathrm{H}$-NMR, MALDI-TOF-MS and IR as shown in Fig. 2 for the ${ }^{1} \mathrm{H}$-NMR data of monomer 2c (top), the ADMET-polymer 3c (middle) and the hydrogenated polymer $4 \mathbf{c}$ (bottom). Successful polymerization of the monomer can be proven by detection of the internal double bonds, which occur during ADMET-polymerization and can be found at $5.3 \mathrm{ppm}$. All other signals become broader and show higher intensity in relation to the terminal double bonds, which are still present after polymerization at $5.8 \mathrm{ppm}$ and 4.9. Thus, this resonance can be used for calculation of the molecular weight by comparison of the ratio of the integrals for the terminal and internal double bonds, which can be determined as $10: 1$ which leads to a molecular weight of $4200 \mathrm{~g} \mathrm{~mol}^{-1}$ in ${ }^{1} \mathrm{H}$-NMR for $3 \mathrm{c}$ and is agreeable with the molecular weight of $3500 \mathrm{~g} \mathrm{~mol}^{-1}$ determined by GPC.

Due to complete insolubility of the final product in common organic solvents complete hydrogenation and deprotection can be demonstrated by ${ }^{1} \mathrm{H}-\mathrm{NMR}$ in $\mathrm{CDCl}_{3}$ only after adding $15 \mathrm{vol} \%$ TFA to the mixture. The characteristic signals for the internal double bonds at $5.3 \mathrm{ppm}$ as well as for the terminal double bond at $5.8 \mathrm{ppm}$ and $4.9 \mathrm{ppm}$ are no longer present in the ${ }^{1} \mathrm{H}$ - 
Table 2 Obtained molecular weights calculated by NMR and GPC, PDI's, yields and trans : cis ratio of the internal double bonds for ADMETpolymerization of the monomers $2 a-2 e$ to polymers $3 a-3 e$ by using different types of catalyst

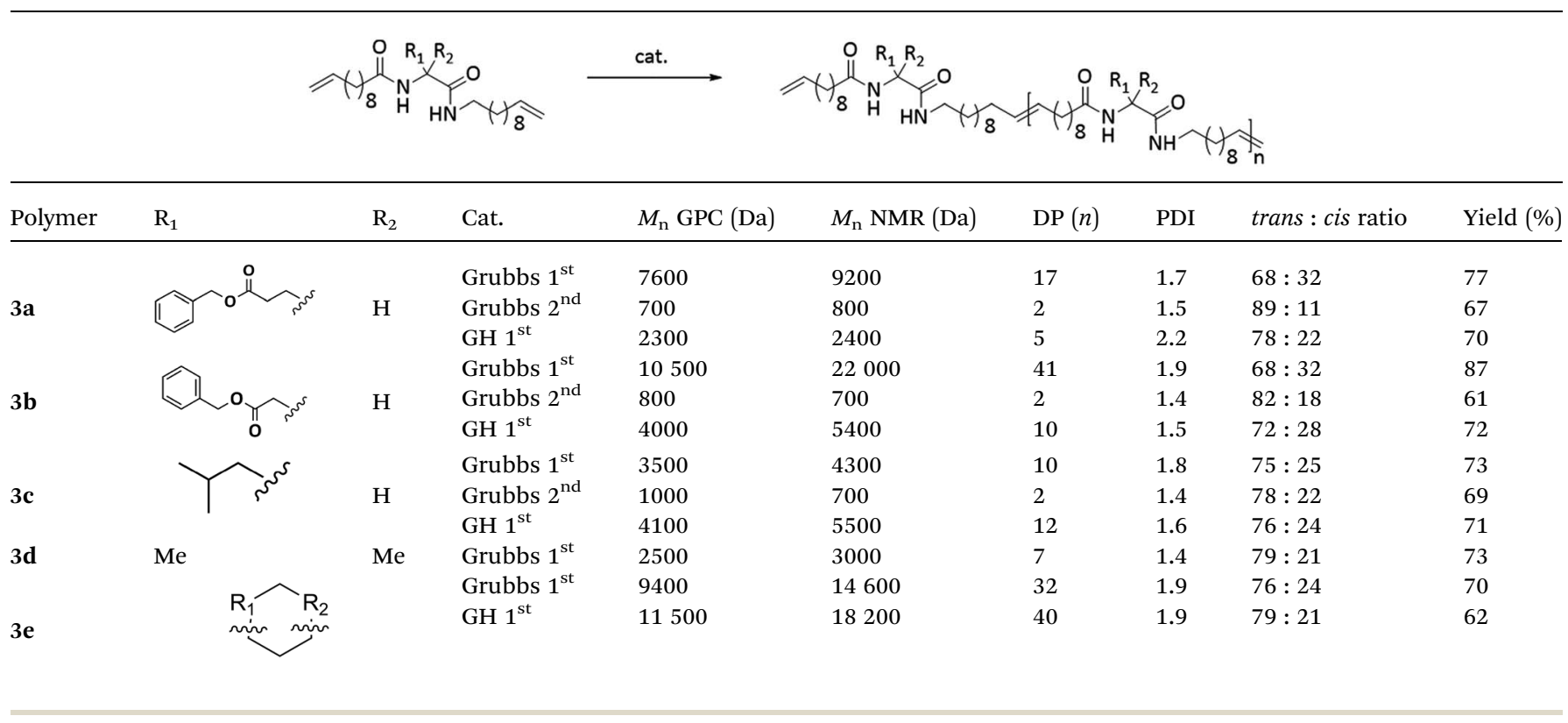

Table 3 Obtained molecular weights calculated by NMR, DP and yields of the unsaturated ADMET polymers $3 a-3 e$ obtained with Grubbs $1^{\text {st }}$ catalyst and saturated polymers $4 \mathrm{a}-4 \mathrm{e}$

\begin{tabular}{llll}
\hline Polymer & $M_{\mathrm{n}}$ NMR (Da) & DP $(n)$ & Yield (\%) \\
\hline 3a & 9200 & 17 & 77 \\
4a & 8400 & 19 & 72 \\
3b & 22000 & 41 & 87 \\
4b & 18500 & 43 & 79 \\
3c & 4300 & 10 & 73 \\
4c & $-a$ & - & 62 \\
3d & 3000 & 7 & 73 \\
4d & 3100 & 7 & 55 \\
3e & 14600 & 32 & 70 \\
4e & 15500 & 33 & 85
\end{tabular}

${ }^{a}$ Calculation of $M_{\mathrm{n}}$ not possible due to overlapping of the methyl end groups and methyl groups in the repeating unit.

NMR spectra of 4c. Furthermore, protons next to the terminal double bonds at $2.0 \mathrm{ppm}$ are disappearing and signals for the methyl groups at $0.9 \mathrm{ppm}$ become broader due to protons of the resulting methyl end group. The successful deprotection of the benzyl-group in the case of $\mathbf{4 a}$ and $\mathbf{4 b}$ can be proven by the disappearance of the signals of the $\mathrm{CH}_{2}$-group next to the aromatic system at $5.1 \mathrm{ppm}$ as well as the characteristic aromatic system signals at 7.3 ppm which is shown in Fig. 3 . New methyl end-group can be detected at $0.9 \mathrm{ppm}$ and was used for calculation of the molecular weight, indicating successful hydrogenation of the double bonds, whereas all other signals are still present showing slightly changed chemical shifts due to interaction with TFA molecules. Degree of polymerization (DP) calculated for the hydrogenated polymers $\mathbf{4 a}, \mathbf{4 b}, \mathbf{4 d}$ and $\mathbf{4 e}$ are always higher as for unsaturated polymers.
All other NMR-spectra of the ADMET- and hydrogenated polymers are shown in the ESI (Fig. S31-S36†). The successful hydrogenation can also be proven in IR-spectroscopy by disappearance of the deformation vibration band of the double bonds at $990 \mathrm{~cm}^{-1}$ and $915 \mathrm{~cm}^{-1}$ in the IR spectrum (Fig. S42S51†).

\section{MALDI-ToF-MS analysis}

In Fig. 4 and 5 MALDi-ToF-MS spectra of the ADMET-polymer 3b and the hydrogenated polymer 4e are shown. All other MALDI-Tof-MS spectra are shown in Fig. S42-S51† with the exception of 3d, $\mathbf{4 a}$ and $\mathbf{4 d}$, which cannot be detected under the chosen conditions. The MS spectra of the ADMET-polymer 3b (Fig. 4) shows a mass distribution from $1091 \mathrm{~g} \mathrm{~mol}^{-1}$ to $4165 \mathrm{~g} \mathrm{~mol}^{-1}$ (Fig. 4a) with a maximum peak at $1604.041 \mathrm{~g}$ $\mathrm{mol}^{-1}$. Two series can be assigned and the distance between signals of the same series amounts to $512 \mathrm{~g} \mathrm{~mol}^{-1}$. The distance between signals of different series is $14 \mathrm{~g} \mathrm{~mol}^{-1}$, in which the signal with the lower molecular weight has always a lower intensity (Fig. 4b). This signal indicates the loss of a $\mathrm{CH}_{2}$-group during ADMET-polymerization due to olefin isomerization, leading to polymers having a slightly different chain length of the alkyl chain, ${ }^{46,54}$ with a small amount of isomerization in e.g. 3b. The low isomerization rate can be proven by the appearance of only one series displaying isomerization products for $\mathbf{3 b}$ and three series for 4e (Fig. 5), whereas high isomerization rates have been observed at cysteine functionalized polymers, resulting in 6 series of isomerized side products. ${ }^{54}$ The signal at $2117 \mathrm{~g} \mathrm{~mol}^{-1}$ can be assigned to the polymer $\left[\mathrm{E}-\mathrm{M}_{2}-\mathrm{EK}\right]^{+}$with potassium as counter ion, which is confirmed by the agreement of the experimental and simulated data (see Fig. 4c). Thus, the obtained main series definitely indicates the expected polymer structure by ADMET polymerization including internal and 


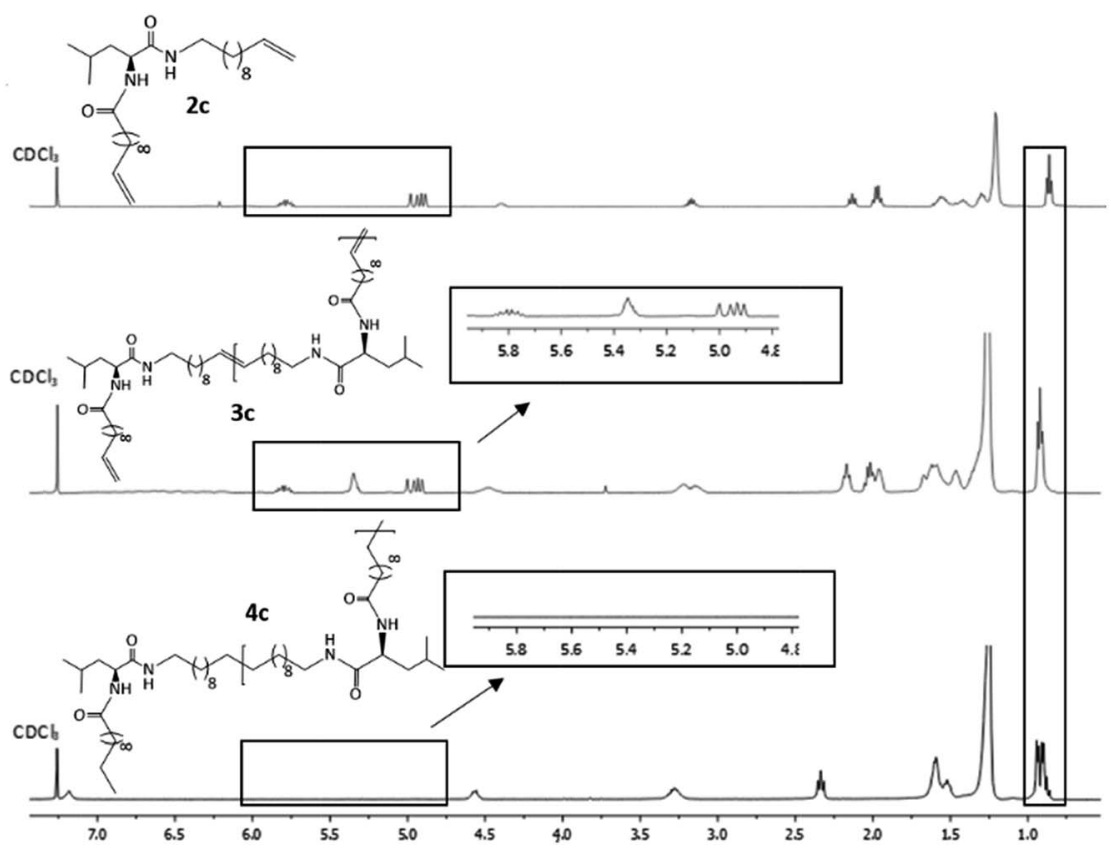

Fig. $2{ }^{1} \mathrm{H}$-NMR spectra of monomer 2c (top), ADMET-polymer 3c (middle) and hydrogenated polymer 4c (bottom) in CDCl 3 . For 4c 15 vol\%. TFA was added.

external double bonds and the loss of ethylene in the repeating unit.

For polymer 4e (Fig. 5) a mass distribution from $937 \mathrm{~g} \mathrm{~mol}^{-1}$ to $2242 \mathrm{~g} \mathrm{~mol}^{-1}$ is obtained, in which the highest absorption peak at $1372 \mathrm{~g} \mathrm{~mol}^{-1}$ indicates the polymer structure $[\mathrm{E}-\mathrm{M}-\mathrm{EK}]^{+}$. Distance between the main series is $434 \mathrm{~g} \mathrm{~mol}^{-1}$, which is in agreement with the molecular weight of the repetitive unit. In addition to the obtained main series up to three different series with a distance of $14 \mathrm{~g} \mathrm{~mol}^{-1}$ can be observed, again indicating the isomerization of the olefin. In comparison to $\mathbf{3 b}$, the isomerization is higher and

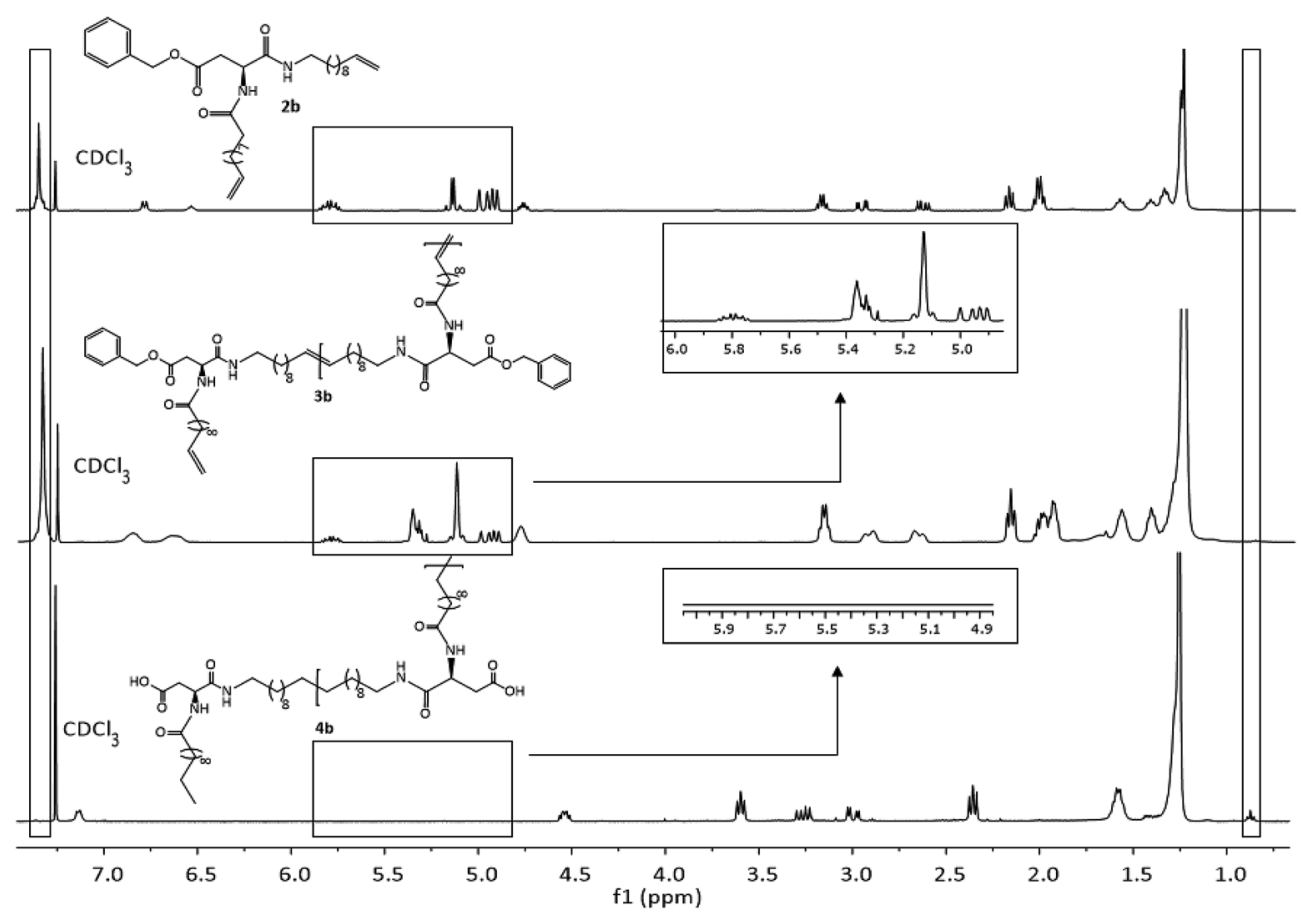

Fig. $3{ }^{1} \mathrm{H}$-NMR spectra of monomer $2 b$ (top), ADMET-polymer $3 b$ (middle) and hydrogenated polymer $4 b$ (bottom) in CDCl 3 . For $4 b$ 15 vol\%. TFA was added. 


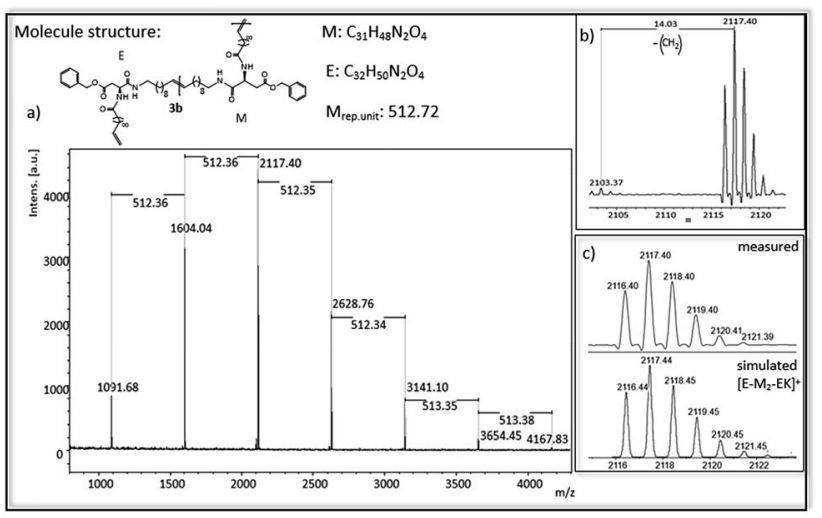

Fig. 4 (a) MALDI-ToF-MS of ADMET polymer 3b (b) zoom in to [E-M ${ }_{2}-$ $E K]^{+}$to show isomerization of the olefin (c) measured and simulated pattern for $\left[\mathrm{E}-\mathrm{M}_{2}-\mathrm{EK}\right]^{+}$.

occurs at more than one site. The main series however proofs the complete hydrogenation of the polymer.

\section{DSC analysis}

The thermal properties of the hydrogenated polymer in comparison to the monomer and the unsaturated polymers were investigated by DSC-measurements. Fig. 6 shows the cooling and heating curve for the monomers $\mathbf{2 a}$ and $\mathbf{2 b}$ (dotted lines) and the polymers $\mathbf{4 a}$ and $\mathbf{4 b}$ (straight lines) after ADMET and hydrogenation.

For monomer 2a melting at $53{ }^{\circ} \mathrm{C}$ and crystallization at $-10{ }^{\circ} \mathrm{C}$ could be observed, whereas monomer $2 \mathbf{b}$ has a significant higher melting and crystallization point $\left(T_{\mathrm{m}}=\right.$ $64.4^{\circ} \mathrm{C} ; T_{\mathrm{c}}=34.7^{\circ} \mathrm{C}$ ). After ADMET-polymerization the investigated compounds $\mathbf{3 a}-\mathbf{3 e}$ become completely amorphous, presumably due to sterically hindrance caused by incorporation of the internal double bonds into the polymer. Crystallization of such polymers strongly depends on the packing of the alkyl chains, which in this case is limited due to the presence of the thus different olefin isomers and the incorporated amino acid in the polymer backbone.

After hydrogenation and deprotection the polymers $\mathbf{4 a}$ and 4b display a crystalline structure as visible by their melting points. In comparison to the corresponding monomer, melting and crystallization of $\mathbf{4 a}$ and $\mathbf{4 b}$ shifts to significantly higher temperatures, in which $\mathbf{4 b}$ has a higher melting and crystallization temperature than 4a. However, in comparison to the unsaturated monomers and polymer $\mathbf{4 a}$, DSC-curves of $\mathbf{4} \mathbf{b}$ are more complex. Melting at $122.8{ }^{\circ} \mathrm{C}$ and $142.9{ }^{\circ} \mathrm{C}$ indicate a melting-recrystallization, which can be due to formation of metastable crystals during the cooling process. ${ }^{55}$ Such crystals are melting first and reorganizing again into more stable areas resulting in an exothermal signal in the DSC curve. These areas are melting later, similar to the behavior found for precision polyolefines with different functional groups acting as defects. ${ }^{\mathbf{5 6}}$ Furthermore, two crystallization temperatures can be observed at $103.6{ }^{\circ} \mathrm{C}$ and $74.4{ }^{\circ} \mathrm{C}$, which may be due to microphase separation. In Table 4 thermal behavior as well as characteristic melting and crystallization data of these samples are summarized.

These observations indicate the big influence of the molecular size and hydrogen-bonding of the amino acids acting as

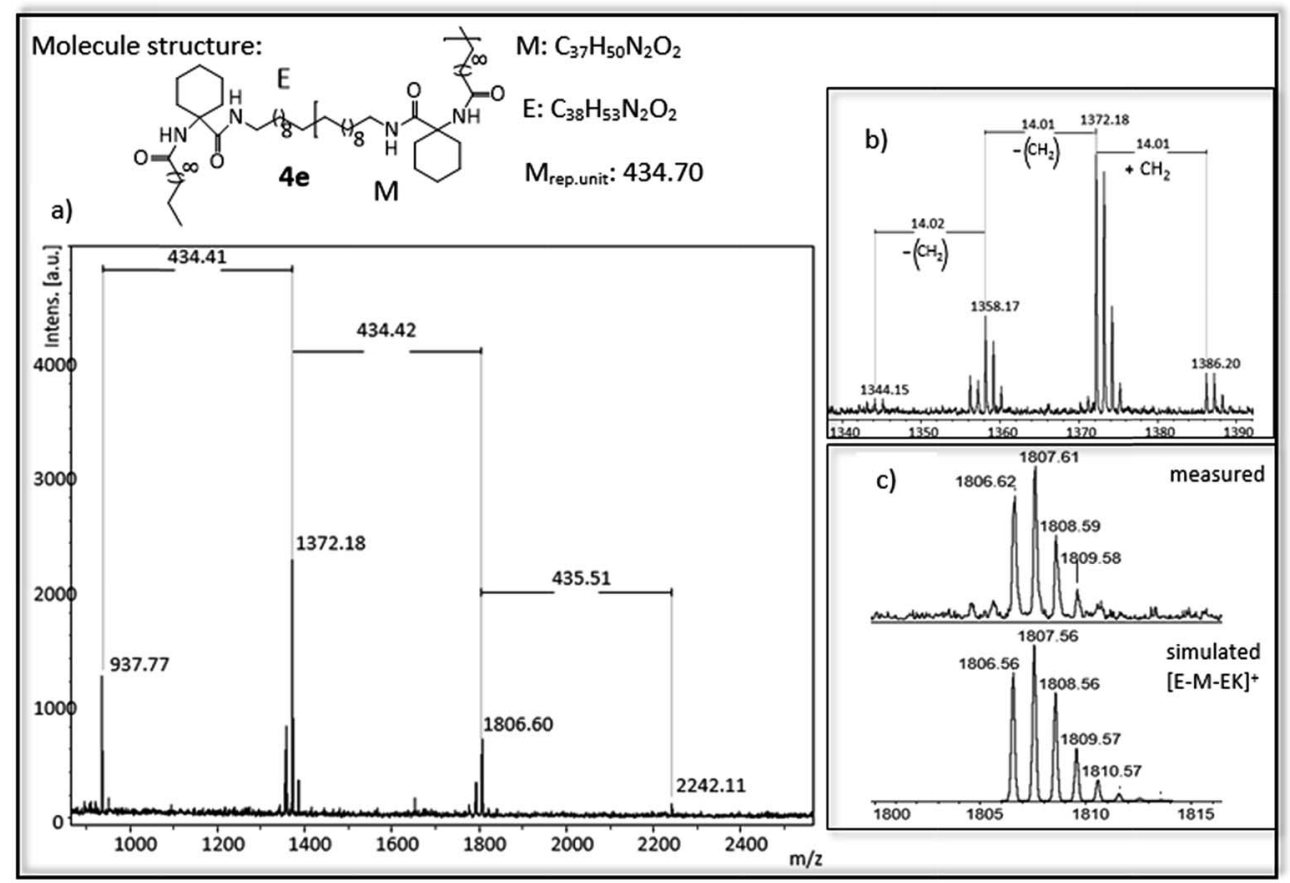

Fig. 5 (a) MALDI-ToF-MS of ADMET polymer 4e (b) zoom in to $[E-M-E K]^{+}$to show isomerization of the olefin (c) measured and simulated pattern for $[E-M-E K]^{+}$. 


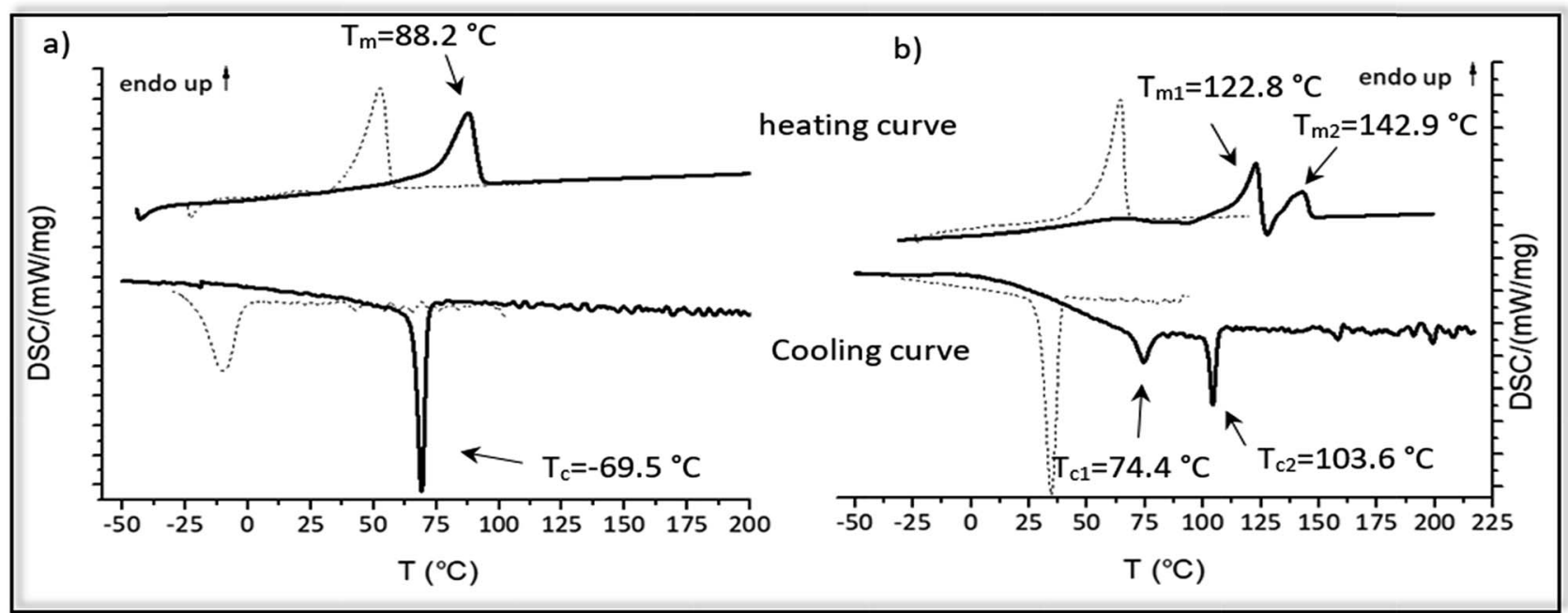

Fig. 6 (a) DSC thermograms of monomer 2a (dotted lines) and the hydrogenated polymer 4a (straight lines) (b) DSC thermograms of monomer $2 \mathrm{~b}$ (dotted lines) and the hydrogenated polymer $4 \mathrm{~b}$ (straight lines).

defects, in which the polymer $\mathbf{4 b}$ bearing aspartic acid after every $19^{\text {th }}$ carbon shows a higher melting and crystallization temperatures than 4a. Such effects of the defect size on the melting and crystallization were reported early e.g. for alkyl branches $^{27}$ and butyl branched polyethylene's and polyphosphoester's. $^{28}$

For all other monomers $2 \mathbf{c}-\mathbf{2 e}$ and polymers $4 \mathbf{c}-\mathbf{4 e}$ no crystalline behavior could be observed as those amino acids with an aliphatic backbone disturbed crystallinity, whereas amino acids with a functional group able to form defined crystal structures. Thus, an additional intermolecular interaction of the carboxylic-acid-defects is proposed for crystallization of the samples, e.g. by dimerization of the carboxylic-acid moieties.

\section{XRD-analysis}

Crystalline structure of polymers $\mathbf{4 a}$ and $\mathbf{4 b}$ was pre-investigated by WAXS measurements (Fig. 7), observing four reflections for both polymers (Table 5). The first reflection in the small angle

Table 4 Thermal behavior of the monomers and polymers with aspartic acid and glutamic acid acting as defects $(2 a-4 a$ and $2 b-4 b)$

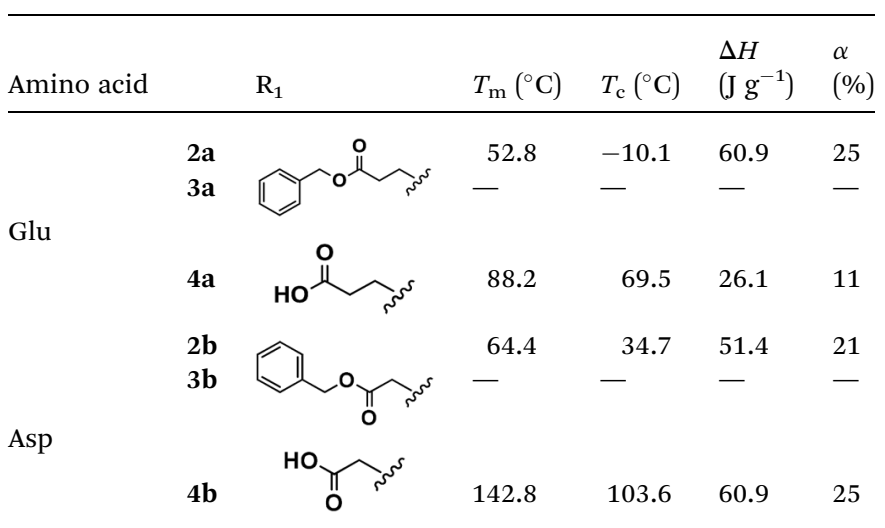

area at a $2 \theta$ of $3.09^{\circ}$ for $\mathbf{4 a}$ and $3.60^{\circ}$ and $\mathbf{4 b}$ is the (001) reflection, indicative of the distance between two amino acid groups along the chain of $2.86 \mathrm{~nm}$ for $\mathbf{4 a}$ and $2.45 \mathrm{~nm}$ for $\mathbf{4 b}$, which is in good agreement with the length of the precision polymers. At almost exactly the doubled value of $2 \theta$ higher order reflection (002) can be detected which was also found for precision sulfone polyethylenes, ${ }^{31}$ demonstrating that the reflection intensity for $\mathbf{4 a}$ is not as intensive as for $\mathbf{4 b}$. These (002) reflections are describing the evolution of a lamellar morphology and both (001) and (002) reflections could have also been observed for other precision PE-polymers of different methylene sequences lengths bearing DAP-units at $16^{\text {th }}, 18^{\text {th }}$ or $20^{\text {th }}$ carbon atom, shifting to higher $2 \theta$ values with decreasing length of the alkyl chain. ${ }^{57}$ Formation of lamellae in $4 a$ could be more hindered than in $\mathbf{4 b}$, caused by the glutamic acid as a major defect, so that the intensity of this reflex for $\mathbf{4 a}$ is significantly smaller than for $\mathbf{4 b}$. As previously reported for ADMET-PE and high density polyethylene, two characteristic reflections at scattering angles of $21.7^{\circ}$ and $24.0^{\circ}$ can be detected, containing information about crystal morphology of the alkyl chains and corresponding to (110) and (200) reflections of a orthorhombic crystal system. ${ }^{58}$ For both polymers $4 \mathbf{a}$ and $\mathbf{4 b}$, broad reflections with maxima at $2 \theta$ of $19.58^{\circ}$ and $21.33^{\circ}$ and $18.98^{\circ}$ and $20.68^{\circ}$ can be observed and thus the crystalline structure is definitely dissimilar to ADMET-PE. ${ }^{19,27}$ The width of the signals can be explained due to the low crystallinity as the defects are large, in accordance with DSC data and literature. ${ }^{27}$ Similar to other precision PE-polymers of different methylene spacer length bearing alkyl moieties ${ }^{19,27}$ chlorines $^{25}$ and metasubstituted aryl ether branches ${ }^{56}$ in the main backbone it is likely that orthorhombic, triclinic and other metastable crystal systems are coexistent as supported by the strong asymmetric distribution of the reflections. ${ }^{19,27}$ Corresponding to the observations for ADMET-polyethylene containing $m$-substituted arylene the amino acid defect presumably can either be excluded from or included into the PE crystals. ${ }^{56}$ 


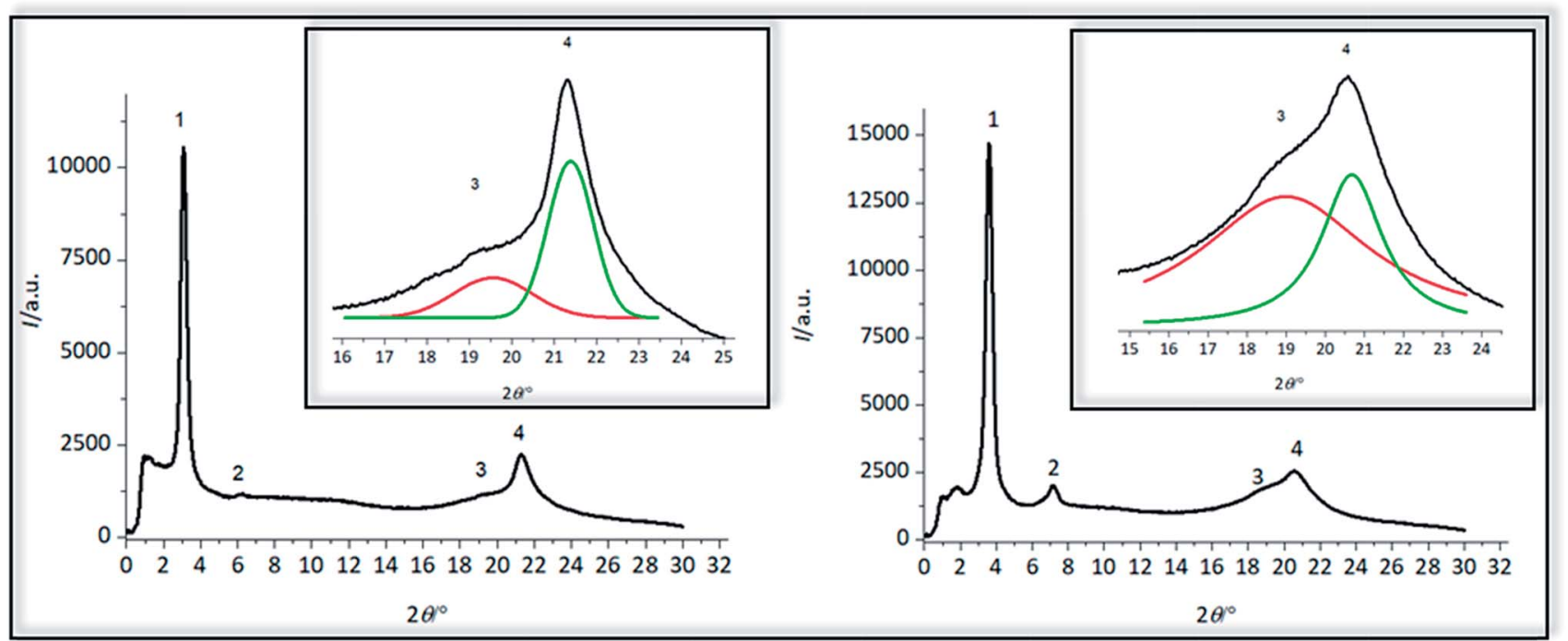

Fig. 7 Wide-angle-X-ray diffraction patterns for precision oligomers $4 a$ and $4 b$.

Table 5 Obtained WAXS data for hydrogenated polymers $4 \mathrm{a}$ and $4 \mathrm{~b}$

\begin{tabular}{llrrr}
\hline Sample & Reflection & $2 \theta /^{\circ}$ & $q / \mathrm{nm}^{-1}$ & $d / \mathrm{nm}$ \\
\hline \multirow{4}{*}{ a } & 1 & 3.09 & 2.20 & 2.86 \\
& 2 & 6.21 & 4.42 & 1.42 \\
& 3 & 19.58 & 13.87 & 0.45 \\
& 4 & 21.33 & 15.10 & 0.42 \\
4b & 1 & 3.60 & 2.56 & 2.45 \\
& 2 & 7.14 & 5.08 & 1.24 \\
& 3 & 18.98 & 13.46 & 0.47 \\
& 4 & 20.68 & 14.64 & 0.43
\end{tabular}

\section{Conclusion}

We here report a strategy for the synthesis and characterization of newly developed precision polyalkyl-polymers with amino acid defects at every $19^{\text {th }} \mathrm{CH}_{2}$ carbon atom. Chiral/achiral as well as polar/nonpolar amino acids were functionalized on the $\mathrm{N}$ - and $\mathrm{C}$-terminus with alkyl branches, bearing terminal double bonds at each end. The synthesis of precision polymers with molecular weights up to $22 \mathrm{kDa}$ was realized by ADMET polymerization under melt-polymerization conditions, followed by complete hydrogenation of the internal double bonds with $p$ toluenesulfonhydrazide $\left(\mathrm{TsNHNH}_{2}\right)$. For ADMET polymerization Grubbs $1^{\text {st }}$, Grubbs $2^{\text {nd }}$ and Grubbs Hoveyda $1^{\text {st }}$ catalyst were tested, the former was found to be the best catalyst in handling and in synthesizing low isomerized, high molecular weight polymers in good yields, proving the structures of the obtained products by ${ }^{1} \mathrm{H}-\mathrm{NMR}$, GPC, MALDI-ToF-Ms and IR. Thermal investigation of the monomers and polymers demonstrated that all compounds having unpolar amino acids (Lleucine, 2-amino-2-methylpropanoic acid, 1-aminocyclohexanecarboxylic acid) in the chain are amorphous, whereas the monomers and the hydrogenated polymers of polar amino acids (glutamic and aspartic acid) show crystalline morphologies. Melting points for glutamic acid modified monomers and polymers are lower in comparison to aspartic acid, demonstrating that the bigger the size of the defect the lower the ability to pack into an ordered structure. The hydrogenated polymer bearing aspartic acid moieties (4b) shows a complex thermal behavior, which is represented by melting-recrystallization and two crystallization points. WAXD-measurements show four reflections for both $\mathbf{4 a}$ and $\mathbf{4 b}$, indicating a distance between the two amino acid groups of $2.86 \mathrm{~nm}$ for $\mathbf{4 a}$ and $2.45 \mathrm{~nm}$ for $\mathbf{4 b}$ along the PE-chain, together with the formation of a lamellar structure. We also assume an orthorhombic crystal in which amino acids can either be excluded from or included into the PE crystals - a final statement however cannot be accomplished on basis of the current data.

\section{Conflicts of interest}

There are no conflicts to declare.

\section{Acknowledgements}

The author grateful acknowledge the grant within the Sonderforschungsbereich SFB TRR 102 (Polymers under multiple constraints) (SR, WHB, Project A03). We also thank Sophie Reimann for MALDI-ToF-MS and Silvio Poppe and Matthias Fischer for providing WAXS measurement.

\section{References}

1 R. M. Michell, A. Mugica, M. Zubitur and A. J. Müller, in Polymer Crystallization I: From Chain Microstructure to Processing, ed. F. Auriemma, G. C. Alfonso and C. de Rosa, Springer International Publishing, Cham, 2017, pp. 215256, DOI: 10.1007/12_2015_327.

2 F. S. Bates, Macromolecules, 1984, 17, 2607-2613.

3 G. Strobl, in The Physics of Polymers Concepts for Understanding Their Structures and Behavior, Springer 
International Publishing, Berlin, Heidelberg, New york, 2007, pp. 215-256, DOI: 10.1007/12_2015_327.

4 E. Haber and C. B. Anfinsen, J. Biol. Chem., 1961, 236, 1361. 5 V. Percec, G. Ungar and M. Peterca, Science, 2006, 313, 55-56. 6 P. Jonkheijm, P. van der Schoot, A. P. H. J. Schenning and E. W. Meijer, Science, 2006, 313, 80-83.

7 B. Heck, T. Hugel, M. Iijima and G. Strobl, Polymer, 2000, 41, 8839-8848.

8 J. Adamcik and R. Mezzenga, Macromolecules, 2012, 45, 1137-1150.

9 I. W. Hamley, Chem. Rev., 2012, 112, 5147-5192.

10 F. J. Baltá-Calleja and R. Hosemann, J. Polym. Sci., Polym. Phys. Ed., 1980, 18, 1159-1165.

11 J. Martinez De Salazar and F. J. BaltáCalleja, J. Cryst. Growth, 1980, 48, 283-294.

12 F. Gutzler and G. Wegner, Colloid Polym. Sci., 1980, 258, 776786.

13 J. M. Salazar and F. J. B. Calleja, Polym. Bull., 1980, 2, 163167.

14 E. Martuscelli and M. Pracella, Polymer, 1974, 15, 306-314.

15 C. L. F. De Ten Hove, J. Penelle, D. A. Ivanov and A. M. Jonas, Nat. Mater., 2004, 3, 33-37.

16 H. Li, L. Caire da Silva, M. D. Schulz, G. Rojas and K. B. Wagener, Polym. Int., 2017, 66, 7-12.

17 P. Atallah, K. B. Wagener and M. D. Schulz, Macromolecules, 2013, 46, 4735-4741.

18 H. Mutlu, L. M. de Espinosa and M. A. R. Meier, Chem. Soc. Rev., 2011, 40, 1404-1445.

19 B. Inci, I. Lieberwirth, W. Steffen, M. Mezger, R. Graf, K. Landfester and K. B. Wagener, Macromolecules, 2012, 45, 3367-3376.

20 G. Lieser, G. Wegner, J. A. Smith and K. B. Wagener, Colloid Polym. Sci., 2004, 282, 773-781.

21 W. Qiu, J. Sworen, M. Pyda, E. Nowak-Pyda, A. Habenschuss, K. B. Wagener and B. Wunderlich, Macromolecules, 2006, 39, 204-217.

22 M. Tasaki, H. Yamamoto, M. Hanesaka, K. Tashiro, E. Boz, K. B. Wagener, C. Ruiz-Orta and R. G. Alamo, Macromolecules, 2014, 47, 4738-4749.

23 P. Kaner, C. Ruiz-Orta, E. Boz, K. B. Wagener, M. Tasaki, K. Tashiro and R. G. Alamo, Macromolecules, 2014, 47, 236245.

24 E. Boz, A. J. Nemeth, K. B. Wagener, K. Jeon, R. Smith, F. Nazirov, M. R. Bockstaller and R. G. Alamo, Macromolecules, 2008, 41, 1647-1653.

25 R. G. Alamo, K. Jeon, R. L. Smith, E. Boz, K. B. Wagener and M. R. Bockstaller, Macromolecules, 2008, 41, 7141-7151.

26 E. Boz, K. B. Wagener, A. Ghosal, R. Fu and R. G. Alamo, Macromolecules, 2006, 39, 4437-4447.

27 G. Rojas, B. Inci, Y. Wei and K. B. Wagener, J. Am. Chem. Soc., 2009, 131, 17376-17386.

28 Y.-R. Zheng, H. T. Tee, Y. Wei, X.-L. Wu, M. Mezger, S. Yan, K. Landfester, K. Wagener, F. R. Wurm and I. Lieberwirth, Macromolecules, 2016, 49, 1321-1330.

29 K. N. Bauer, H. T. Tee, I. Lieberwirth and F. R. Wurm, Macromolecules, 2016, 49, 3761-3768.
30 M. Pulst, M. H. Samiullah, U. Baumeister, M. Prehm, J. Balko, T. Thurn-Albrecht, K. Busse, Y. Golitsyn, D. Reichert and J. Kressler, Macromolecules, 2016, 49, 6609-6620.

31 T. W. Gaines, E. B. Trigg, K. I. Winey and K. B. Wagener, Macromol. Chem. Phys., 2016, 217, 2351-2359.

32 T. W. Baughman and K. B. Wagener, in Metathesis Polymerization, ed. M. R. Buchmeiser, Springer Berlin Heidelberg, Berlin, Heidelberg, 2005, pp. 1-42, DOI: 10.1007/b101318.

33 F. Sanda and T. Endo, Macromol. Chem. Phys., 1999, 200, 2651-2661.

34 H. Kudo, F. Sanda and T. Endo, Macromolecules, 1999, 32, 8370-8375.

35 F. Sanda, T. Abe and T. Endo, J. Polym. Sci., Part A: Polym. Chem., 1997, 35, 2619-2629.

36 J. Morcellet-Sauvage, M. Morcellet and C. Loucheux, Makromol. Chem., 1981, 182, 949-963.

37 J. Morcellet-Sauvage, M. Morcellet and C. Loucheux, Makromol. Chem., 1982, 183, 821-829.

38 H. D. Maynard, S. Y. Okada and R. H. Grubbs, J. Am. Chem. Soc., 2001, 123, 1275-1279.

39 H. D. Maynard, S. Y. Okada and R. H. Grubbs, Macromolecules, 2000, 33, 6239-6248.

40 J. K. Kammeyer, A. P. Blum, L. Adamiak, M. E. Hahn and N. C. Gianneschi, Polym. Chem., 2013, 41, 3929-3933.

41 R. B. Breitenkamp, Z. Ou, K. Breitenkamp, M. Muthukumar and T. Emrick, Macromolecules, 2007, 40, 7617-7624.

42 T. E. Hopkins, J. H. Pawlow, D. L. Koren, K. S. Deters, S. M. Solivan, J. A. Davis, F. J. Gómez and K. B. Wagener, Macromolecules, 2001, 34, 7920-7922.

43 F. N. Behrendt and H. Schlaad, Polym. Chem., 2017, 8, 366369.

44 J. K. Leonard, T. E. Hopkins, K. Chaffin and K. B. Wagener, Macromol. Chem. Phys., 2008, 209, 1485-1494.

45 K. M. Lambert, J. M. Bobbitt, S. A. Eldirany, L. E. Kissane, R. K. Sheridan, Z. D. Stempel, F. H. Sternberg and W. F. Bailey, Chem.-Eur. J., 2016, 22, 5156-5159.

46 V. I. Petkovska, T. E. Hopkins, D. H. Powell and K. B. Wagener, Anal. Chem., 2006, 78, 3624-3631.

47 F. C. Courchay, J. C. Sworen, I. Ghiviriga, K. A. Abboud and K. B. Wagener, Organometallics, 2006, 25, 6074-6086.

48 S. E. Lehman and K. B. Wagener, Macromolecules, 2002, 35, 48-53.

49 Y. Schrodi, Aldrichimica Acta, 2007, 40, 45.

50 S. Kobayashi, L. M. Pitet and M. A. Hillmyer, J. Am. Chem. Soc., 2011, 133, 5794-5797.

51 Z.-L. Li, L. Li, X.-X. Deng, L.-J. Zhang, B.-T. Dong, F.-S. Du and Z.-C. Li, Macromolecules, 2012, 45, 4590-4598.

52 E. B. Berda and K. B. Wagener, Macromolecules, 2008, 41, 5116-5122.

53 S. Reimann, U. Baumeister and W. H. Binder, Macromol. Chem. Phys., 2014, 215, 1929.

54 V. I. Petkovska, T. E. Hopkins, D. H. Powell and K. B. Wagener, Macromolecules, 2005, 38, 5878-5885.

55 S. Song, W. Miao, Z. Wang, D. Gong and Z.-R. Chen, Polymer, 2015, 64, 76-83. 
56 S.-F. Song, Y.-T. Guo, R.-Y. Wang, Z.-S. Fu, J.-T. Xu and Z.-Q. Fan, Macromolecules, 2016, 49, 6001-6011.

57 S. Reimann, V. Danke, M. Beiner and W. H. Binder, J. Polym. Sci., Part A: Polym. Chem., 2017, 55, 3736-3748.
58 M. F. Butler, A. M. Donald, W. Bras, G. R. Mant, G. E. Derbyshire and A. J. Ryan, Macromolecules, 1995, 28, 6383-6393. 